\title{
Varieties of apolar subschemes of toric surfaces
}

\author{
Matteo Gallet, Kristian Ranestad and Nelly Villamizar
}

\begin{abstract}
Powersum varieties, also called varieties of sums of powers, have provided examples of interesting relations between varieties since their first appearance in the 19th century. One of the most useful tools to study them is apolarity, a notion originally related to the action of differential operators on the polynomial ring. In this work, we make explicit how one can see apolarity in terms of the Cox ring of a variety. In this way, powersum varieties are a special case of varieties of apolar schemes; we explicitly describe examples of such varieties in the case of two toric surfaces, when the Cox ring is particularly well-behaved.
\end{abstract}

\section{Introduction}

Powersum varieties, parametrizing expressions of a form as a sum of powers of linear polynomials, provide examples of surprising relations between varieties, namely between them and the hypersurfaces defined by the forms. These varieties have been widely studied since the 19th century: Sylvester considered and solved the case of binary forms (see [22] and [23]). A number of further cases have been treated more recently, see [12], [15], [16], [18], [19] and [20].

A powersum variety $\operatorname{VSP}(f, k)$ is associated to a polynomial $f \in \operatorname{Sym}^{d} V$ on a vector space $V$ over a field $K$, and to a positive integer number $k$. It is defined as the Zariski closure in the Hilbert scheme of subschemes of $\mathbb{P}(V)$ of length $k$ of the set

$$
\left\{\left[\left[l_{1}\right], \ldots,\left[l_{k}\right]\right] \in \operatorname{Hilb}_{k} \mathbb{P}(V): f=l_{1}^{d}+\cdots+l_{k}^{d},\right.
$$

$$
\text { where } \left.\left[l_{i}\right] \in \mathbb{P}(V) \text { are pairwise distinct }\right\} \text {. }
$$

Although $K$ in this definition can be an arbitrary field, throughout this paper we always consider it to be the field of complex numbers $\mathbb{C}$.

Key words and phrases: toric surfaces, apolarity, apolar schemes, powersum varieties. 2010 Mathematics Subject Classification: 14M25, 14J99, 14 N99. 
Powersum varieties are special cases of a more general construction: given a projective variety $X \subseteq \mathbb{P}^{n}$, let $y \in \mathbb{P}^{n}$ be a general point and $k$ be the minimal integer such that there are $k$ points in $X$ whose span contains $y$. Thus, $k$ is the smallest integer such that the $(k-1)$-th secant variety of $X$ fills the space $\mathbb{P}^{n}$. Define $\operatorname{VPS}_{X}(y, k)$ to be the closure in $\operatorname{Hilb}_{k}(X)$ of the set of smooth subschemes of length $k$ whose span contains $y$. If $X$ is a Veronese variety, then one may interpret $y$ as the class $[f]$ of a homogeneous form $f$ of some degree $d$. The $k$-tuples of points on $X$ whose span contains $[f]$ represent expressions of $f$ as a sum of $d$-th powers of linear forms, hence in this way we recover the notion of powersum variety of $f$ and $k$. Therefore, if $X \subseteq \mathbb{P}\left(\operatorname{Sym}^{d} V\right)$ is the $d$-uple embedding of $\mathbb{P}(V)$, then $\operatorname{VSP}(f, k)=$ $\operatorname{VPS}_{X}([f], k)$.

Furthermore, $\operatorname{VPS}_{X}(y, k)$ can be seen as the "Variety of aPolar Subschemes":

Definition 1.1. A subscheme $Z \subseteq X$ is called apolar to $y \in \mathbb{P}^{n}$, if $y$ is contained in the linear span of $Z$ in $\mathbb{P}^{n}$.

Note that $\operatorname{VPS}_{X}(y, k)$ contains only those apolar subschemes that are in the closure of the set of smooth apolar subschemes. When $\operatorname{dim} X>3$, then the general point $y$ may have singular apolar subschemes of length $k$ that do not belong to this closure.

Apolar schemes have been studied, in the classical setting of powersum varieties, considering the ideal of differential operators annihilating a given homogeneous form. More precisely, one considers two polynomial rings $S=\mathbb{C}\left[x_{0}, \ldots, x_{n}\right]$ and $T=\mathbb{C}\left[y_{0}, \ldots, y_{n}\right]$, and the action of the variables $y_{i}$ on $x_{j}$ defined by differentiation: $y_{i} \cdot x_{j}=\partial x_{j} / \partial x_{i}$. In this way, for a homogeneous polynomial $f$ of degree $d$, one defines the set $H_{f} \subseteq T$ of differential operators of degree $d$ annihilating $f$. Then $H_{f}$ is a hyperplane in the vector space $\mathbb{C}\left[y_{0}, \ldots, y_{n}\right]_{d}$ and a scheme $Z$ is apolar to $[f]$ if and only if $I_{Z, d} \subseteq H_{f}$.

In this paper, we generalize the notion of apolarity and investigate $\operatorname{VPS}_{X}(y, k)$ using the Cox ring $\operatorname{Cox}(X)$ of $X$, namely the $\mathbb{C}$-algebra of sections of all line bundles on $X$, graded by the Picard group $\operatorname{Pic}(X)$. If $X$ is a toric variety, $\operatorname{Cox}(X)$ has a simple structure, namely it is a polynomial ring (see [3]): we use this fact to explore VPS $_{X}$ in particular cases of toric surfaces.

Let $T=\bigoplus_{A \in \operatorname{Pic}(X)} T_{A}$ be $\operatorname{Cox}(X)$, and for each $A \in \operatorname{Pic}(X)$ let $S_{A}$ be the space of linear forms on $T_{A}$, i.e. $S_{A}=T_{A}^{*}$. If $A$ is very ample on $X$, then its global sections $T_{A}$ define the embedding $\nu_{A}: X \hookrightarrow \mathbb{P}\left(S_{A}\right)$. To every subscheme $Z \subseteq X$ we associate an ideal $I_{Z} \subseteq T$ :

$$
I_{Z}:=\bigoplus_{B \in \operatorname{Pic}(X)} I_{Z, B} \subseteq T \text { and } I_{Z, B}:=\left\{g \in T_{B}:\left.g\right|_{Z} \equiv 0\right\} \text {. }
$$


Definition 1.1 may be generalized as follows:

Definition 1.2. For any nonzero $f \in S_{A}$ let $H_{f} \subseteq T_{A}$ be the hyperplane of sections that vanish at the point $[f] \in \mathbb{P}\left(S_{A}\right)$. A subscheme $Z \subseteq X$ is called apolar to $f \in S_{A}$ if $I_{Z, A} \subseteq H_{f}$.

One of the facts that makes the classical theory of apolarity such a powerful tool is that it makes possible to translate the previous condition of containment of vector spaces into a condition involving ideals. This fact can be generalized as follows. For each $B \in \operatorname{Pic}(X)$ define

$$
I_{f, B}=\left\{\begin{array}{l}
H_{f}: T_{A-B}=\left\{g \in T_{B}: g \cdot T_{A-B} \subseteq H_{f}\right\}, \quad \text { if } A-B>0 \\
T_{B}, \quad \text { otherwise, }
\end{array}\right.
$$

where $A-B>0$ if the line bundle $A-B$ has global sections, and set

$$
I_{f}=\bigoplus_{B \in \operatorname{Pic}(X)} I_{f, B} \subseteq T \text {. }
$$

The classical apolarity lemma (see [11, Lemma 1.15]) can be read as follows.

Lemma 1.3. For a subscheme $Z \subseteq X$ and $f \in S_{A}$, then $I_{Z} \subseteq I_{f}$ if and only if $I_{Z, A} \subseteq H_{f}$.

Proof. It suffices to show the if-direction of the equivalence. If $B>A$ then $I_{f, B}=T_{B}$, so it suffices to consider $B<A$. But $I_{Z, B} \cdot T_{A-B} \subseteq I_{Z, A} \subseteq H_{f}$ implies $I_{Z, B} \subseteq$ $H_{f}: T_{A-B}=I_{f, B}$ and the lemma follows.

Additionally, we rephrase in terms of the Cox ring the maps associating to a polynomial all its partial derivatives of a given order: for $A, B \in \operatorname{Pic}(X)$ and $f \in S_{A}$ we define the linear map

$$
\phi_{f, B}: T_{B} \longrightarrow S_{A-B} \quad \text { and } \quad g \longmapsto g(f)
$$

such that

$$
\begin{gathered}
g(f)\left(g^{\prime}\right)=g^{\prime} g(f) \in \mathbb{C} \quad \text { for } \quad g^{\prime} \in T_{A-B} \quad \text { i.e. } \\
H_{g(f)}:=\left(H_{f}:\langle g\rangle\right) \subseteq T_{A-B} .
\end{gathered}
$$

Notice that $\operatorname{ker} \phi_{f, B}=I_{f, B}$.

The previous generalization of apolarity was also recently considered in [10] in the particular case of smooth toric varieties. The author uses the apolarity lemma to prove upper bounds on the minimum length of subschemes whose linear span contains a general point.

In this paper, we present three examples where we describe $\operatorname{VPS}_{X}\left([f], r_{f}\right)$ where $X$ is a toric surface different from the projective plane, $f$ a general section 
in $S_{A}$ for some $A \in \operatorname{Pic}(X)$ and $r_{f}$ the minimal integer $r$ such that $\operatorname{VPS}_{X}([f], r)$ is not empty. In Section 2, we set up the theory for apolarity in the case $X=\mathbb{P}^{1} \times \mathbb{P}^{1}$. We split the proof of the following theorem among Sections 3, 4 and 5 .

Theorem 1.4. Let $X$ be a projective variety, $A \in \operatorname{Pic}(X)$ and $f \in S_{A}$ be a general section.

(A) If $X=\mathbb{P}^{1} \times \mathbb{P}^{1}$ and $A=(2,2)$, then $\operatorname{VPS}_{\mathbb{P}^{1} \times \mathbb{P}^{1}}([f], 4)$ is a threefold isomorphic to a smooth linear complex in the Grassmannian $G(2,4)$ blown up along a rational normal quartic curve.

(B) If $X=\mathbb{P}^{1} \times \mathbb{P}^{1}$ and $A=(3,3)$, then $\operatorname{VPS}_{\mathbb{P}^{1} \times \mathbb{P}^{1}}([f], 6)$ is isomorphic to a smooth Del Pezzo surface of degree 5 .

(C) If $X=F_{1}$, namely the blow up of $\mathbb{P}^{2}$ in one point embedded as a cubic scroll in $\mathbb{P}^{4}$, and $A=3 H$ where $H$ is the hyperplane class of $F_{1}$, then $\operatorname{VPS}_{F_{1}}([f], 8)$ is isomorphic to $\mathbb{P}^{2}$ blown up in 8 points.

Apolar rational or elliptic curves play a crucial role in our arguments, particularly in the use of the following facts. For rational curves Sylvester showed (see $[22])$ :

Lemma 1.5. Let $C \subseteq \mathbb{P}^{2 d-1}$ be a rational normal curve of degree $2 d-1$, then there is a unique $d$-secant $\mathbb{P}^{d-1}$ to $C$ passing through a general point, i.e. $\operatorname{VPS}_{C}(y, d) \cong\{p t\}$ for a general point $y \in \mathbb{P}^{2 d-1}$. Let $C \subseteq \mathbb{P}^{2 d}$ be a rational normal curve of degree $2 d$ and $y$ a general point in $\mathbb{P}^{2 d-1}$, then $\operatorname{VPS}_{C}(y, d+1) \cong C$.

For elliptic curves, the following lemma follows from Room's description of determinantal varieties. We give a proof in Lemma 5.3 in Section 5.

Lemma 1.6. Let $C \subseteq \mathbb{P}^{2 d-2}$ be an elliptic normal curve of degree $2 d-1$ and $y$ a general point in $\mathbb{P}^{2 d-1}$, then $\operatorname{VPS}_{C}(y, d) \cong C$.

\section{Apolarity for $\mathbb{P}^{1} \times \mathbb{P}^{1}$}

Let us consider $X=\mathbb{P}^{1} \times \mathbb{P}^{1}$. In this case, the Picard group of $X$ is $\mathbb{Z}^{2}$ and its Cox ring is $T=\mathbb{C}\left[t_{0}, t_{1}\right]\left[u_{0}, u_{1}\right]$, see for instance [4, Example 5.2.2].

We can write $T=\bigoplus_{a, b \in \mathbb{Z}} T_{a, b}$, where $T_{a, b}$ is the set of bihomogeneous polynomials of bidegree $(a, b)$. In this case, setting $S_{a, b}=T_{a, b}^{*}$, the group $S=\bigoplus_{a, b \in \mathbb{Z}} S_{a, b}$ has the structure of a ring. In fact, $S=\mathbb{C}\left[x_{0}, x_{1}\right]\left[y_{0}, y_{1}\right]$ where the duality between homogeneous components of $S$ and $T$ is induced by differentiation: $t_{i}=\partial / \partial x_{i}$ and $u_{i}=\partial / \partial y_{i}$. If $f \in S_{a, b}$ and the annihilator is defined as

$$
f_{c, d}^{\perp}=\left\{g \in T_{c, d}: g(f)=0\right\},
$$


then $f_{a, b}^{\perp}=H_{f}$, where $H_{f}$ is the hyperplane from Definition 1.2. Setting $f_{c, d}^{\perp}=$ $f_{a, b}^{\perp}: T_{(a-c, b-d)}$, the annihilator ideal of $f$ and the ideal $I_{f}$ defined in (1) coincide:

$$
f^{\perp}:=\{g \in T: g(f)=0\}=I_{f} .
$$

When $a, b>0$, the divisors of class $(a, b)$ on $\mathbb{P}^{1} \times \mathbb{P}^{1}$ determine the Segre-Veronese embedding

$$
\begin{aligned}
\nu_{a, b}: \quad \mathbb{P}^{1} \times \mathbb{P}^{1} & \hookrightarrow \mathbb{P}^{a b+a+b} \\
\left(\left[l_{1}\right],\left[l_{2}\right]\right) & \mapsto \quad\left[l_{1}^{a} l_{2}^{b}\right]
\end{aligned}
$$

where $l_{1} \in\left\langle x_{0}, x_{1}\right\rangle, l_{2} \in\left\langle y_{0}, y_{1}\right\rangle$, and $\mathbb{P}^{a b+a+b}$ is identified with $\mathbb{P}\left(S_{a, b}\right)$. We sometimes call $\nu_{a, b}$ the $(a, b)$-embedding.

A subscheme $\Gamma \subseteq \mathbb{P}^{1} \times \mathbb{P}^{1}$ is apolar to $f$ if $I_{\Gamma,(a, b)} \subseteq f_{a, b}^{\perp}$, or equivalently $[f] \in$ $\operatorname{span} \nu_{a, b}(\Gamma)$. The variety of apolar schemes $\operatorname{VPS}_{\mathbb{P}^{1} \times \mathbb{P}^{1}}([f], r)$ may be interpreted as a variety of sums of powers, i.e. as the Zariski closure of

$$
\left\{\left[\left(\left[l_{11}\right],\left[l_{21}\right]\right), \ldots,\left(\left[l_{1 r}\right],\left[l_{2 r}\right]\right)\right] \in \operatorname{Hilb}_{r}\left(\mathbb{P}^{1} \times \mathbb{P}^{1}\right): f=\sum_{i=1}^{r} l_{1 i}^{a} l_{2 i}^{b}\right\} .
$$

As in the standard homogeneous case, the minimal $r$ such that $\operatorname{VPS}_{\mathbb{P}^{1} \times \mathbb{P}^{1}}([f], r)$ is nonempty, is called the rank of $f$, denoted $\operatorname{rank}(f)$.

Let us remark that a general form in $S_{a, b}$ has rank $r$ if and only if $r$ is the minimal $k$ such that the $k$-secant variety of the Segre-Veronese coincides with $\mathbb{P}^{a b+a+b}$.

The computation of the dimension of secant varieties carried in [2, Corollary 2.3 , implies that if $f$ is a bihomogeneous general form of bidegree $(a, b)$, then

$$
\operatorname{rank}(f)= \begin{cases}2 d+2 & \text { if }(a, b)=(2,2 d) \text { for some } d \\ \left\lceil\frac{(a+1)(b+1)}{3}\right] & \text { otherwise. }\end{cases}
$$

For such a general form $f$, the dimension of $\operatorname{VPS}_{\mathbb{P}^{1} \times \mathbb{P}^{1}}([f], r)$ is determined by $\operatorname{rank}(f)$ as described in the following proposition (see [5, Proposition 3.2] for the classical case).

Proposition 2.1. Let $f \in S_{a, b}$ be a general bihomogeneous form of rank $r$. Then $\operatorname{VPS}_{\mathbb{P}^{1} \times \mathbb{P}^{1}}([f], r)$ is an irreducible variety of dimension

$$
\operatorname{dim} \operatorname{VPS}_{\mathbb{P}^{1} \times \mathbb{P}^{1}}([f], r)=\left\{\begin{array}{cl}
3 & \text { if }(a, b)=(2,2 d), \\
3\left(\left\lceil\frac{(a+1)(b+1)}{3}\right\rceil-\frac{(a+1)(b+1)}{3}\right) & \text { if }(a, b) \neq(2,2 d) .
\end{array}\right.
$$


Proof. Let us denote $\operatorname{Hilb}_{r}\left(\mathbb{P}^{1} \times \mathbb{P}^{1}\right)$ by $\mathcal{H}$. We consider the incidence variety

$$
\mathcal{X}=\left\{([\Gamma],[f]) \in \mathcal{H} \times \mathbb{P}^{a b+a+b}:[\Gamma] \in \operatorname{VPS}_{\mathbb{P}^{1} \times \mathbb{P}^{1}}([f], r)\right\} .
$$

Then we have the two projection maps:

$$
\pi_{1}: \mathcal{X} \longrightarrow \mathcal{H} \text { and } \pi_{2}: \mathcal{X} \longrightarrow \mathbb{P}^{a b+a+b}
$$

Let $\mathcal{U}$ be the open subset of $\mathcal{H}$ parametrizing zero-dimensional schemes given by $r$ distinct points in $\mathbb{P}^{1} \times \mathbb{P}^{1}$. It is possible to restrict $\mathcal{U}$ so that the $(a, b)$-th powers of all linear forms associated to such points are linearly independent. In this way, we can prove that $\pi_{1}$ is dominant. Moreover, if $[\Gamma] \in \mathcal{U}$, the fiber of $\pi_{1}$ over $[\Gamma]$ is an open set of a linear space of dimension $r-1$. Since $\mathcal{H}$ is irreducible [7], then also $\mathcal{X}$ is irreducible and of dimension $3 r-1$. The fiber of $\pi_{2}$ over $[f] \in \mathbb{P}^{a b+a+b}$ is $\operatorname{VPS}_{\mathbb{P}^{1} \times \mathbb{P}^{1}}([f], r)$, so for a general $f$, the variety $\operatorname{VPS}_{\mathbb{P}^{1} \times \mathbb{P}^{1}}([f], r)$ has dimension $3 r-1-(a b+a+b)$. Using formula (4) the statement follows.

\section{Bihomogeneous forms of bidegree $(2,2)$}

The Segre-Veronese embedding (3) is in this case the $(2,2)$-embedding of $\mathbb{P}^{1} \times \mathbb{P}^{1}$ in $\mathbb{P}^{8}$, denoted $\nu_{2,2}: \mathbb{P}^{1} \times \mathbb{P}^{1} \hookrightarrow \mathbb{P}^{8}$. If $f$ is a general bihomogeneous form in $S_{2,2}$, then by the formula of the rank (4) applied to the case $(a, b)=(2,2 d)$ with $d=1$, we have $\operatorname{rank}(f)=4$, and $\operatorname{dim} \operatorname{VPS}_{\mathbb{P}^{1} \times \mathbb{P}^{1}}([f], 4)=3$ by Proposition 2.1 .

Lemma 3.1. For a general form $f \in S_{2,2}$ the orthogonal $f^{\perp}$ is generated by $f_{2,1}^{\perp}$, $f_{1,2}^{\perp}, f_{3,0}^{\perp}$ and $f_{0,3}^{\perp}$. Moreover, both $f_{2,1}^{\perp}$ and $f_{1,2}^{\perp}$ have dimension 4 .

Proof. Since $\operatorname{dim} T_{2,1}=6$ and $\operatorname{dim} S_{0,1}=2$, and $f$ is general, the $\operatorname{ker} \phi_{f,(2,1)}=$ $f_{2,1}^{\perp}$ has dimension 4 , with $\phi_{f,(2,1)}$ as defined in (2). By symmetry, also $f_{1,2}^{\perp}$ has dimension 4 .

Consider the vector subspace $T_{0,1} \cdot f_{2,1}^{\perp} \subseteq f_{2,2}^{\perp}$ : if it is of dimension 8, it means that we do not need elements from $f_{2,2}^{\perp}$ to generate $f^{\perp}$. Suppose that $\operatorname{dim} T_{0,1}$. $f_{2,1}^{\perp}<8$ : if $g_{1}, \ldots, g_{4}$ is a basis for $f_{2,1}^{\perp}$, then $u_{0} g_{1}, \ldots, u_{0} g_{4}, u_{1} g_{1}, \ldots, u_{1} g_{4}$ are linearly dependent. So, for some $h_{1}, h_{2} \in f_{2,1}^{\perp}$ we have $u_{0} h_{1}+u_{1} h_{2}=0$. Then $h_{1}=$ $u_{1} \tilde{h}$ and $-h_{2}=u_{0} \tilde{h}$ for some nonzero $\tilde{h} \in T_{0,2}$. Hence $T_{0,1} \cdot \tilde{h} \subseteq f^{\perp}$, which forces $\tilde{h} \in$ $f^{\perp}$. But $\tilde{h}$ has bidegree $(2,0)$, and by the generality assumption on $f$ there is no nontrivial element in $f_{2,0}^{\perp}$. Hence $\operatorname{dim} T_{0,1} \cdot f_{2,1}^{\perp}=8$, and so $T_{0,1} \cdot f_{2,1}^{\perp}=f_{2,2}^{\perp}$.

Moreover, since $f$ is a form of bidegree $(2,2)$, then $f_{a, b}^{\perp}=T_{a, b}$ whenever $a$ or $b$ is greater than or equal to 3 . Notice that $T_{3, b}=T_{3,0} \cdot T_{0, b}$, and $T_{a, 3}=T_{0,3} \cdot T_{a, 0}$, for every $a, b \geq 1$. Thus, $f_{2,1}^{\perp}, f_{1,2}^{\perp}$ together with $f_{3,0}^{\perp}=T_{3,0}$ and $f_{0,3}^{\perp}=T_{0,3}$ generate the ideal $f^{\perp}$. 
The space of sections $f_{2,1}^{\perp}$ defines a linear system of $(2,1)$-curves on $\mathbb{P}^{1} \times \mathbb{P}^{1}$ and, by Lemma 3.1 , a rational map $\delta_{2,1}: \mathbb{P}^{1} \times \mathbb{P}^{1} \longrightarrow \mathbb{P}^{3}$ fitting in the diagram

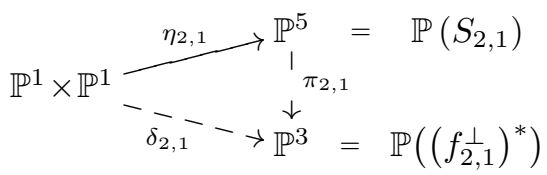

where $\eta_{2,1}$ is the map induced by the complete linear systems of $(2,1)$-curves in $\mathbb{P}^{1} \times \mathbb{P}^{1}$, and $\pi_{2,1}$ is a linear projection. All constructions and results from now on apply to both $f_{2,1}^{\perp}$ and $f_{1,2}^{\perp}$.

First, we prove that $\delta_{2,1}$ is a morphism. For this, we analyze the projection center $L_{2,1}$ of $\pi_{2,1}$. By definition, $L_{2,1} \subseteq \mathbb{P}\left(S_{2,1}\right)$ is spanned by the forms annihilated by $f_{2,1}^{\perp}$, i.e. by the partials $\partial f / \partial y_{0}$ and $\partial f / \partial y_{1}$. Consider the surface scroll $Y_{2,1}:=$ $\eta_{2,1}\left(\mathbb{P}^{1} \times \mathbb{P}^{1}\right) \subseteq \mathbb{P}\left(S_{2,1}\right)$. The $(1,0)$-curves on $\mathbb{P}^{1} \times \mathbb{P}^{1}$ are mapped to lines in $Y_{2,1}$, while the $(0,1)$-curves are mapped to conics. The planes of these conics are the planes spanned by forms $q\left(x_{0}, x_{1}\right) \cdot l\left(y_{0}, y_{1}\right) \in S_{2,1}$, where $l\left(y_{0}, y_{1}\right) \in\left\langle y_{0}, y_{1}\right\rangle$ is a fixed linear form. We let $W_{2,1}$ be the threefold union of these planes.

Lemma 3.2. Let $f \in S_{2,2}$ be a general form. Then no linear combination of its partial derivatives $\partial f / \partial y_{0}$ and $\partial f / \partial y_{1}$ is of the form $q\left(x_{0}, x_{1}\right) \cdot l\left(y_{0}, y_{1}\right)$, where $q$ and $l$ are respectively a quadratic and a linear form. In particular, the line $L_{2,1} \subseteq \mathbb{P}\left(S_{2,1}\right)$ does not intersect $W_{2,1}$.

Proof. Write $f$ as $y_{0}^{2} Q+y_{0} y_{1} Q^{\prime}+y_{1}^{2} Q^{\prime \prime}$. Then, a linear combination $\lambda \partial f / \partial y_{0}+$ $\mu^{\partial f / \partial y_{1}}$ is of the form $q \cdot l$ if and only if $\lambda Q+\mu Q^{\prime}$ is proportional to $\lambda Q^{\prime}+\mu Q^{\prime \prime}$. Since $f$ is general, we can suppose that $Q, Q^{\prime}$ and $Q^{\prime \prime}$ are linearly independent. Then the two pencil of quadrics $\lambda Q+\mu Q^{\prime}$ and $\lambda Q^{\prime}+\mu Q^{\prime \prime}$ have at most one point in common, which hence must coincide with $Q^{\prime}$. But

$$
\lambda Q+\mu Q^{\prime}=Q^{\prime} \Longleftrightarrow \lambda=0 \text { and } \lambda Q^{\prime}+\mu Q^{\prime \prime}=Q^{\prime} \Longleftrightarrow \mu=0 .
$$

This proves the claim.

Corollary 3.3. The map $\delta_{2,1}$ defined by $f_{2,1}^{\perp}$ is a morphism. Moreover, all lines in $Z_{2,1}:=\delta_{2,1}\left(\mathbb{P}^{1} \times \mathbb{P}^{1}\right)$ are linear projections of lines in $Y_{2,1}=\eta_{2,1}\left(\mathbb{P}^{1} \times \mathbb{P}^{1}\right)$. In particular, no conic in $Y_{2,1}$ is mapped to a line by $\pi_{2,1}$. The analogous result holds for the $(1,2)$ case.

Proof. Since the projection center $L_{2,1}$ does not intersect $W_{2,1} \supseteq Y_{2,1}$, the projection $\pi_{2,1}$ restricted to $Y_{2,1}$ and hence $\delta_{2,1}$ are morphisms, and no conic in $Y_{2,1}$ is mapped to a line in $Z_{2,1}$. Furthermore, $f_{1,1}^{\perp}=\{0\}$, so $L_{2,1}$ does not lie in the span of any $(1,1)$-curve in $Y_{2,1}$; therefore, every line in $Z_{2,1}$ is the linear projection of a $(1,0)$-curve, i.e. a line in $Y_{2,1}$. 
Lemma 3.4. Let $f \in S_{2,2}$ be a general form and suppose that $g_{1}, g_{2} \in f_{2,1}^{\perp}$ span a linear space of dimension 2. Then, either the ideal $\left(g_{1}, g_{2}\right)$ defines a scheme of length 4 in $\mathbb{P}^{1} \times \mathbb{P}^{1}$, or the pencil of $(2,1)$-curves defined by $g_{1}$ and $g_{2}$ has a common component, a (1,0)-curve.

Proof. We need to exclude that the pencil of $(2,1)$-curves defined by $g_{1}$ and $g_{2}$ has a fixed component that is a $(2,0)$, a $(1,1)$ or a $(0,1)$-curve. We treat these cases one by one.

If there is a common $(2,0)$-curve, then all curves in the pencil split into it and a $(0,1)$-line. Therefore, there exists $q \in \mathbb{C}\left[u_{0}, u_{1}\right]_{2}$ such that

$$
\left(\alpha t_{0}+\beta t_{1}\right) q \cdot f=0 \quad \forall \alpha, \beta \in \mathbb{C},
$$

so $q \in f_{0,2}^{\perp}$, but this contradicts Lemma 3.1. Similarly, if we assume that there is a common $(1,1)$-curve, then $f_{1,1}^{\perp}$ would be non-trivial and this again contradicts Lemma 3.1.

We are left with the case when the pencil $\left\langle g_{1}, g_{2}\right\rangle$ has a $(0,1)$-curve $\ell$ in its base locus. Consider the maps in the diagram (5), with $L_{2,1}$ the center of the projection $\pi_{2,1}$. Then $\ell$ is sent to a conic by $\eta_{2,1}$. Hence there is a pencil of hyperplanes in $\mathbb{P}^{5}$ passing through $L_{2,1}$ and having a conic in its base locus. Since the base locus of a pencil of hyperplanes in $\mathbb{P}^{5}$ is a $\mathbb{P}^{3}$, both the conic and the line $L_{2,1}$ lie in a $\mathbb{P}^{3}$. The latter happens if and only if $L_{2,1}$ intersects the plane spanned by such a conic, but this is not possible by Corollary 3.3.

The image $Z_{2,1}$ of $\mathbb{P}^{1} \times \mathbb{P}^{1}$ under $\delta_{2,1}$ is a rational scroll, since it is rational and covered by the images of the lines in the scroll $\eta_{2,1}\left(\mathbb{P}^{1} \times \mathbb{P}^{1}\right)$. We describe its singular locus.

Lemma 3.5. $Z_{2,1}$ is a quartic surface in $\mathbb{P}^{3}$ that has double points along a twisted cubic curve and no triple points.

Proof. A scheme $z$ of length 3 in $\mathbb{P}^{1} \times \mathbb{P}^{1}$ is contained in a $(1,1)$-curve $C$, so if $z$ is mapped to a point by $\delta_{2,1}=\pi_{2,1} \circ \eta_{2,1}$ the projection center $L_{2,1}$ is contained in the span of $\eta_{2,1}(z) \subseteq Y_{2,1}$, and hence in the span of the image of $C$ in $Y_{2,1}$. But then $C$ is mapped to a line in $Z_{2,1}$, and this is excluded by Corollary 3.3. Therefore, $Z_{2,1}$ has no triple points. A general plane section of $Z_{2,1}$ is the image of a smooth rational quartic curve in $Y_{2,1}$; therefore, it is a rational quartic curve, and so has 3 singular points that span a plane. Hence $\operatorname{Sing}\left(Z_{2,1}\right)$ spans $\mathbb{P}^{3}$ and is a cubic curve. From the double point formula (see [9, Theorem 9.3]) we see that the double point locus of the restriction $\left.\pi_{2,1}\right|_{Y_{2,1}}$ is a curve on $Y_{2,1}$ of degree 6 because it is linearly equivalent to $-K_{Y_{2,1}}$ (the anticanonical divisor of $\left.Y_{2,1}\right)$. If $\operatorname{Sing}\left(Z_{2,1}\right)$ is 
not a twisted cubic, then it has to contain a line. The preimage under $\pi_{2,1}$ of such a line is then either a conic $C$ or two skew lines $E_{1}$ and $E_{2}$. In the first case, the center $L_{2,1}$ of $\pi_{2,1}$ intersects $\operatorname{span}(C)$, but this is not possible because of Lemma 3.2. In the second case, we have $L_{2,1} \subseteq \operatorname{span}\left(E_{1} \cup E_{2}\right)$. Since $\operatorname{span}\left(E_{1} \cup E_{2}\right) \cong$ $\mathbb{P}^{3}$, there is a pencil of hyperplanes in $\mathbb{P}^{5}$ containing it; each of them intersects $Y_{2,1}$ in $E_{1} \cup E_{2}$ and in a residual conic $D$. Hence we obtain a pencil of conics $D$ such that $\operatorname{span}(D)$ intersects $\operatorname{span}\left(E_{1} \cup E_{2}\right)$ in a line $F$. In this way, we get a pencil of lines $F$ in $\operatorname{span}\left(E_{1} \cup E_{2}\right)$; such pencil fills a quadric in $\operatorname{span}\left(E_{1} \cup E_{2}\right)$, and therefore the center $L_{2,1}$ intersects this quadric in 2 points; this situation is again ruled out by Lemma 3.2. Therefore, the only possibility left is that $\operatorname{Sing}\left(Z_{2,1}\right)$ is a twisted cubic.

Remark 3.6. Consider a smooth scheme $[\Gamma] \in \operatorname{VPS}_{\mathbb{P}^{1} \times \mathbb{P}^{1}}([f], 4)$ apolar to $f$, namely $I_{\Gamma} \subseteq f^{\perp}$. Notice that the dimension of $I_{\Gamma,(2,1)}$ equals the number of linearly independent planes in $\mathbb{P}^{3}$ passing through $\delta_{2,1}(\Gamma)$. Moreover $2 \leq \operatorname{dim} I_{\Gamma,(2,1)} \leq 3$, where the latter inequality follows since $\delta_{2,1}$ is defined on the whole $\mathbb{P}^{1} \times \mathbb{P}^{1}$. Lemma 3.5 excludes that the dimension of $I_{\Gamma,(2,1)}$ is 3 , since in that case we would have $\delta_{2,1}(\Gamma)=\{\mathrm{pt}\}$. Hence $\operatorname{dim} I_{\Gamma,(2,1)}=2$ and $\delta_{2,1}(\Gamma)$ spans a line.

Remark 3.6 yields a rational map defined on smooth apolar schemes:

$$
\begin{aligned}
\Phi_{2,1}: \operatorname{VPS}_{\mathbb{P}^{1} \times \mathbb{P}^{1}}([f], 4) & -\rightarrow G\left(2, f_{2,1}^{\perp}\right) \\
{[\Gamma] } & \mapsto I_{\Gamma,(2,1)}
\end{aligned}
$$

Lemma 3.7. For a general bihomogeneous form $f$ of bidegree $(2,2)$, the rational map $\Phi_{2,1}$ in (6) extends to a morphism on the whole $\operatorname{VPS}_{\mathbb{P}^{1} \times \mathbb{P}^{1}}([f], 4)$. Let $D_{2,1}$ be the curve

$$
D_{2,1}=\left\{[\ell] \in G\left(2, f_{2,1}^{\perp}\right): \ell \subseteq Z_{2,1}\right\} \quad \text { where } \quad Z_{2,1}=\delta_{2,1}\left(\mathbb{P}^{1} \times \mathbb{P}^{1}\right) .
$$

Then the fiber over a point $p$ under $\Phi_{2,1}$ is a smooth rational curve if $p \in D_{2,1}$ and it is at most one point when $p \notin D_{2,1}$. In particular, $\Phi_{2,1}$ is birational.

Proof. Since being collinear (see Remark 3.6) is a closed property, $\Phi_{2,1}$ extends to the closure of smooth apolar schemes, namely to $\operatorname{VPS}_{\mathbb{P}^{1} \times \mathbb{P}^{1}}([f], 4)$.

Let $[\Gamma] \in \operatorname{VPS}_{\mathbb{P}^{1} \times \mathbb{P}^{1}}([f], 4)$ and let $\ell_{\Gamma}$ be the line in $\mathbb{P}^{3}$ containing $\delta_{2,1}(\Gamma)$. If $\left[\ell_{\Gamma}\right] \notin D_{2,1}$, then $\ell_{\Gamma} \cap Z_{2,1}$ is a scheme of length 4 , namely it is $\delta_{2,1}(\Gamma)$. Hence the fiber over $\ell_{\Gamma}$ is exactly $[\Gamma]$. Therefore, if $\ell \subseteq \mathbb{P}^{3}$ is any line not contained in $Z_{2,1}$, then either $[\ell]$ is not in the image of $\Phi_{2,1}$, or it is the image of exactly one scheme in $\operatorname{VPS}_{\mathbb{P}^{1} \times \mathbb{P}^{1}}([f], 4)$.

Let $\ell \subseteq \mathbb{P}^{3}$ be a line contained in $Z_{2,1}$. Denote by $\ell^{\prime}$ the $(1,0)$-line in $\mathbb{P}^{1} \times \mathbb{P}^{1}$ such that $\delta_{2,1}\left(\ell^{\prime}\right)=\ell$. Since the preimage of $\operatorname{Sing}\left(Z_{2,1}\right)$ under the projection is linearly 
equivalent to the anticanonical divisor of $Y$, and so every line in $Y_{2,1}$ intersects it in two points, then $\ell$ intersects $\operatorname{Sing}\left(Z_{2,1}\right)$ in 2 points. In fact, by Corollary 3.3, every line in $Z_{2,1}$ is a projection of a line in $Y$. We know that the preimage of $\ell \cap \operatorname{Sing}\left(Z_{2,1}\right)$ under $\delta_{2,1}$ consists of a scheme of length 4 that intersects $\ell^{\prime}$ in a subscheme of length 2. Summing up, $\delta_{2,1}^{-1}(\ell)=\ell^{\prime} \cup\left\{z_{\ell}\right\}$, where $z_{\ell}$ is a scheme of length 2 that is mapped to $\ell$ by $\delta_{2,1}$.

Let $\Gamma \subseteq \mathbb{P}^{1} \times \mathbb{P}^{1}$ be an apolar scheme such that $\delta_{2,1}(\Gamma)$ is contained in $\ell$. Since $\Gamma$ has length 4 and $\Gamma \subseteq \delta_{2,1}^{-1}(\ell)$, then the span of $\eta_{2,1}(\Gamma)$ must contain $\ell^{\prime}$. On the other hand, since $\Gamma$ is apolar to $f$, it is also apolar to both $\partial f / \partial y_{0}$ and $\partial f / \partial y_{1}$. Therefore, the span of $\eta_{2,1}(\Gamma)$ contains the line $L_{2,1}$, the center of the projection $\pi_{2,1}$ in diagram (5). This rules out the case $\operatorname{span} \Gamma \cong \mathbb{P}^{1}$ and $\operatorname{span} \Gamma \cong \mathbb{P}^{2}$, since in both cases the center of the projection would intersect $Y_{2,1}$, contradicting Corollary 3.3. Hence $\eta_{2,1}(\Gamma)$ spans a $\mathbb{P}^{3}$ in $\mathbb{P}^{5}$, and therefore there is a length 2 subscheme of $\Gamma$ that is not contained in $\ell^{\prime}$. Clearly, this subscheme coincides with $z_{\ell}$.

Let us consider a plane in $\mathbb{P}^{3}$ through $\ell$. By construction of the map $\ell$, such plane defines (up to scalars) a form $g \in f_{2,1}^{\perp}$ that factors as $g=l \tilde{g}$, where $l$ is a $(1,0)$-form whose vanishing locus in $\mathbb{P}^{1} \times \mathbb{P}^{1}$ is $\ell^{\prime}$. If $g$ vanishes on a scheme $\Gamma$ of length 4 , that is apolar to $f$ and is mapped to $\ell$ by $\delta_{2,1}$, then $z_{\ell} \subseteq \Gamma$ and $\tilde{g}$ must vanish on the length 2 subscheme $z_{\ell}$. In fact, there is a pencil of $(1,1)$-forms vanishing on $z_{\ell}$ that together with $l$ vanish on $\Gamma$. Therefore every subscheme in the fiber over a $\Phi_{2,1}(\Gamma)$ contains $z_{\ell}$ and is contained in the reducible curve $\ell^{\prime} \cup Z(\tilde{g})$.

The set of apolar subschemes of length 4 in $\ell^{\prime} \cup Z(\tilde{g})$ are described in the following lemma.

Lemma 3.8. Let $g \in f_{2,1}^{\perp}$ such that $g=l \tilde{g}$ for forms $l$ and $\tilde{g}$ of bidegree $(1,0)$ and $(1,1)$ respectively. Then the zero locus $C_{g}$ of $g$ supports two pencils of length 4 apolar schemes. One pencil has a common subscheme of length 2 on $Z(\tilde{g})$ and a moving subscheme of length 2 on $Z(l)$, while the subschemes of the other pencil has a unique ) common point on $Z(l)$, and a moving subscheme of length 3 on $Z(\tilde{g})$.

Proof. The fact that $g$ is apolar to $f$ means that the point $[f]$ is contained in the span of the $(2,2)$-embedding of $C_{g}$. To avoid redundant notation, we also denote by $C_{g}$ the curve $\nu_{2,2}\left(C_{g}\right)$. By construction, $C_{g}$ splits as $C_{g}=C_{1} \cup C_{2}$ where $C_{1}$ is a conic and $C_{2}$ is a quartic rational normal curve. Notice that $\operatorname{span} C_{1} \cong \mathbb{P}^{2}$ and $\operatorname{span} C_{2} \cong \mathbb{P}^{4}$. Consider the projection from $[f]$, denoted by $\rho: \operatorname{span} C_{g} \cong \mathbb{P}^{6}-\rightarrow$ $\mathbb{P}^{5}$. Notice that $\left.\rho\right|_{\text {span } C_{1}}$ and $\left.\rho\right|_{\text {span } C_{2}}$ are isomorphisms, because otherwise $C_{1}$ or $C_{2}$ will be apolar to $f$ which contradicts Lemma 3.1. Set $P$ to be the preimage under $\rho$ of the line $\rho\left(\operatorname{span} C_{1}\right) \cap \rho\left(\operatorname{span} C_{2}\right)$, and define the lines $L_{1}=P \cap \operatorname{span} C_{1}$ and $L_{2}=P \cap \operatorname{span} C_{2}$. 
Consider the line $L_{1}$ : by construction, it passes through $Q=C_{1} \cap C_{2}$, the only singularity of $C_{g}$, and intersects the conic $C_{1}$ in another point $T$. The line through $[f]$ and $T$ is contained in the plane $P$, thus intersects $L_{2}$ in a point $\widetilde{T}$. Since $C_{2}$ is a smooth rational quartic, the set

$$
\left\{\text { trisecant planes of } C_{2} \text { passing through } \widetilde{T}\right\} \text {. }
$$

corresponds to the variety of sum of powers of a quartic bivariate form decomposed into three summands, and by Lemma 1.5 it is isomorphic to $\mathbb{P}^{1}$. If we pick the three points of intersection of such a trisecant plane with $C_{2}$ and we add the point $T$, we obtain four points whose span contains $[f]$. Thus, we have constructed a $\mathbb{P}^{1}$ of schemes of length 4 apolar to $f$ constituted of 3 points lying on $C_{2}$, and one common point lying on $C_{1}$.

Consider the line $L_{2}$ : by construction, it passes through the singularity $Q$, and it intersects the secant variety of $C_{2}$ (a cubic threefold) in another point $R$. The line through $[f]$ and $R$ is contained in the plane $P$, thus intersects $L_{1}$ in a point $\widetilde{R}$. Since $R$ is in the secant variety of $C_{2}$, there exist two points in $C_{2}$ whose span contains $R$. Moreover, there is a pencil of lines in span $C_{1}$ passing through $\widetilde{R}$, which defines a pencil of length 2 schemes on $C_{1}$. So we get a pencil of length 4 schemes apolar to $f$, all of them with a length 2 scheme on $C_{2}$ in common.

To complete the proof of Lemma 3.7 we apply Lemma 3.8 to $\ell^{\prime} \cup Z(\tilde{g})$ : only the pencil of apolar subschemes with a fixed subscheme of length 2 on $Z(\tilde{g})$ is mapped to $\ell$ by $\delta_{2,1}$.

Lemma 3.9. Let $g \in f_{2,1}^{\perp}$ such that $g=l_{1} l_{2} \tilde{l}$ for forms $l_{i}$ of bidegree $(1,0)$ and $\tilde{l}$ of bidegree $(0,1)$. Then the zero locus $C_{g}$ of $g$ supports three pencils of length 4 apolar schemes. Two of them are fibers of $\Phi_{2,1}$, while $\Phi_{2,1}$ maps the third isomorphically to a line in $G\left(2, f_{2,1}^{\perp}\right)$.

Proof. The first part of the proof follows a similar argument as that in Lemma 3.8, so we only provide a sketch. The $(2,2)$-embedding of $C_{g}$ splits into three conics $C_{1}, C_{2}$ and $\widetilde{C}$, such that $C_{1} \cap \widetilde{C}=\left\{Q_{1}\right\}, C_{2} \cap \widetilde{C}=\left\{Q_{2}\right\}$ and $C_{1} \cap C_{2}=\varnothing$.

By projecting from $[f]$ one can prove that there exists a plane $P$ such that $P \cap \operatorname{span} C_{1}=\ell_{1}, P \cap \operatorname{span}\left(\widetilde{C} \cup C_{2}\right)=\ell_{2}$ where $\ell_{1}, \ell_{2}$ are lines, and $P$ contains the line through $[f]$ and $Q_{1}$. The line $\ell_{1}$ meets $C_{1}$ in $Q$ and in another point $R_{1}$. The line through $R_{1}$ and $[f]$ meets $\ell_{2}$ in a point $T$. By a similar argument but projecting from $T$, we obtain a point $R_{2} \in C_{2}$ and a pencil of pairs of points $R_{3}, R_{4} \in \widetilde{C}$ such that $T$ is in $\operatorname{span}\left(\left\{R_{2}, R_{3}, R_{4}\right\}\right)$. It follows that $[f]$ belongs to $\operatorname{span}\left(\left\{R_{1}, R_{2}, R_{3}, R_{4}\right\}\right)$. In this way, we find a pencil of apolar schemes with $R_{1}$ and $R_{2}$ as fixed points, one on each of the curves $C_{1}$ and $C_{2}$, and a moving part of length 2 on $\widetilde{C}$. On $Z_{2,1}$, the 
curves $C_{1}$ and $C_{2}$ are mapped to lines by $\delta_{2,1}$, while $\widetilde{C}$ is mapped to a conic. Their union is a plane section of $Z_{2,1}$, and the apolar schemes are all collinear. Since $R_{1}$ and $R_{2}$ are mapped to the same point $S$, this pencil of apolar schemes must lie on the pencil of lines through $S$. Therefore, the image of this pencil in $G\left(2, f_{2,1}^{\perp}\right)$ is a line. Two other pencils of such schemes with mobile parts supported on $C_{1}$ and $C_{2}$ can be constructed similarly. Each of the latter two pencils is mapped to a line in $Z_{2,1}$, namely the images of $C_{1}$ and $C_{2}$, and is therefore, by Lemma 3.7 , the fiber of the morphism $\Phi_{2,1}$ over a point in $D_{1,2}$. This concludes the proof.

Proposition 3.10. For a general bihomogeneous form $f$ of bidegree $(2,2)$ the image of the map $\Phi_{2,1}$ defined in (6) is a smooth linear section of the Grassmannian $G\left(2, f_{2,1}^{\perp}\right)$.

Proof. Since $\operatorname{dim} \operatorname{VPS}_{\mathbb{P}^{1} \times \mathbb{P}^{1}}([f], 4)=3, \operatorname{dim} G\left(2, f_{2,1}^{\perp}\right)=4$ and $\Phi_{2,1}$ is birational onto its image, the image is a hypersurface $U$ in $G\left(2, f_{2,1}^{\perp}\right)$.

The degree 3 component of the Chow group of $G\left(2, f_{2,1}^{\perp}\right)$ is generated freely by one Schubert class $\Sigma_{1}$, so $[U]=d \Sigma_{1}$ for some $d$. The intersection of an $\alpha$-plane $\Sigma_{2}$ with $\Sigma_{1}$ gives the only class $\Sigma_{3}$ in degree 1 in the Chow group. Hence $[U] \cdot \Sigma_{2}=d \Sigma_{3}$. We prove that $d=1$.

Let us consider the intersection of $U$ with an $\alpha$-plane $\Sigma_{2}$. Every $\alpha$-plane in $G\left(2, f_{2,1}^{\perp}\right)$ is of the form $\Sigma_{2}(g)=\left\{\left\langle g_{1}, g_{2}\right\rangle \subseteq f_{2,1}^{\perp}: g \in\left\langle g_{1}, g_{2}\right\rangle\right\}$ for some $g \in f_{2,1}^{\perp}$. On the other hand, such a form $g$ defines a rational curve $C_{g}$, and its $(2,2)$-embedding in $\mathbb{P}^{8}$ has degree 6 . Therefore, the intersection of $U$ with $\Sigma_{2}(g)$ has preimage under $\Phi_{2,1}$ given by

$$
\begin{aligned}
\Phi_{2,1}^{-1}\left(U \cap \Sigma_{2}(g)\right) & =\left\{[\Gamma] \in \operatorname{VPS}_{\mathbb{P}^{1} \times \mathbb{P}^{1}}([f], 4): g \in I_{\Gamma,(2,1)}\right\} \\
& =\left\{[\Gamma] \in \operatorname{VPS}_{\mathbb{P}^{1} \times \mathbb{P}^{1}}([f], 4): \Gamma \subseteq C_{g}\right\} .
\end{aligned}
$$

If $C_{g}$ is smooth, then by Lemma 1.5 we derive that $\Phi_{2,1}^{-1}\left(U \cap \Sigma_{2}(g)\right) \cong \mathbb{P}^{1}$.

Consider now the case when $C_{g}$ is not smooth. If $C_{g} \subseteq \mathbb{P}^{1} \times \mathbb{P}^{1}$ splits into the union of a line and a smooth conic (intersecting in a point), then $\nu_{2,2}\left(C_{g}\right) \subseteq \mathbb{P}^{8}$ splits into a conic $C_{1}$ and a quartic $C_{2}$, both rational and smooth. In this case, $\Phi_{2,1}^{-1}\left(U \cap \Sigma_{2}(g)\right)$ has two irreducible components, both rational and smooth, by Lemma 3.8. We claim that these are the only two components of maximal dimension of the scheme $\Phi_{2,1}^{-1}\left(U \cap \Sigma_{2}(g)\right)$. In fact, if there were $[\Gamma] \in \operatorname{VPS}_{\mathbb{P}^{1} \times \mathbb{P}^{1}}([f], 4)$ such that $\nu_{2,2}(\Gamma) \subseteq C_{1}$, then the conic $C_{1}$ would be apolar to $f$; this would imply that there is a nonzero element in $f_{1,0}^{\perp}$, contradicting Lemma 3.1. An analogous argument excludes the possibility that $\nu_{2,2}(\Gamma) \subseteq C_{2}$. On the other hand, there is at most one scheme $\Gamma$ formed by three points on $C_{1}$ and one point $B$ on $C_{2}$. In fact, by construction $[f] \in \operatorname{span} \Gamma$, thus the line through $[f]$ and $B$ intersects $\mathbb{P}^{2}=\operatorname{span} C_{1}$, so 
it is contained in the plane $P$ from Lemma 3.8. Since $P \cap C_{2}$ is the singular point $Q$, such line coincides with the line through $[f]$ and $Q$, and that means that we have at most one scheme $\Gamma$ of this kind.

If $C_{g} \subseteq \mathbb{P}^{1} \times \mathbb{P}^{1}$ splits into the union of three lines, then $\nu_{2,2}\left(C_{g}\right) \subseteq \mathbb{P}^{8}$ splits into three conics $C_{1}, C_{2}$ and $\widetilde{C}$. In this case, $\Phi_{2,1}^{-1}\left(U \cap \Sigma_{2}(g)\right)$ has three irreducible components, all rational and smooth, but, by Lemma 3.9, only one of them is not contracted by $\Phi_{2,1}$.

Therefore, as the $\alpha$-planes vary, we obtain a family of smooth and rational curves. Hence the only possibility is that $U$ is a linear complex, i.e. a hyperplane section of $G\left(2, f_{2,1}^{\perp}\right)$.

Let $X_{2,1}$ be the image of $\Phi_{2,1}$, we show that $X_{2,1}$ is smooth. Assume by contradiction that $X_{2,1}$ is singular. Then it contains two families of planes. In particular, it contains a family of $\alpha$-planes as planes in $G\left(2, f_{2,1}^{\perp}\right)$. But any $\alpha$-plane in $G\left(2, f_{2,1}^{\perp}\right)$ is of the form $\Sigma_{2}(g)$ and intersects $X_{2,1}$ in a curve, so cannot be contained in $X_{2,1}$. This proves the claim.

Proposition 3.11. Every $[\Gamma] \in \operatorname{VPS}_{\mathbb{P}^{1} \times \mathbb{P}^{1}}([f], 4)$ is apolar to $f$.

Proof. Let $[\Gamma] \in \operatorname{VPS}_{\mathbb{P}^{1} \times \mathbb{P}^{1}}([f], 4)$, we have to show that $I_{\Gamma} \subseteq f^{\perp}$. By Lemma 3.7, both $I_{\Gamma} \cap f_{2,1}^{\perp}$ and $I_{\Gamma} \cap f_{1,2}^{\perp}$ are two-dimensional. If both $I_{\Gamma,(2,1)}$ and $I_{\Gamma,(1,2)}$ are twodimensional, then $I_{\Gamma} \subseteq f^{\perp}$. Suppose now that $I_{\Gamma,(2,1)}$ has dimension 3: then, by Lemma 3.4, the system of $(2,1)$-curves in $I_{\Gamma} \cap f_{2,1}^{\perp}$ must have a common component, a $(1,0)$-line $\ell$. Suppose first that $\ell$ is a common component of the whole system $I_{\Gamma,(2,1)}$. Then the residual 3 -dimensional family of $(1,1)$-curves can have at most one point in common. Therefore, the line $\ell$ contains a length 3 subscheme of $\Gamma$. The image of $\ell$ under $\delta_{1,2}$ is a conic, but this contradicts the fact that $\delta_{1,2}(\Gamma)$ is collinear. Suppose next that $\ell$ is not a common component of the linear system $I_{\Gamma,(2,1)}$. Then $I_{\Gamma,(2,1)}=\left\langle g_{1}, g_{2}, g_{3}\right\rangle$ with $g_{3} \notin I_{\Gamma} \cap f_{2,1}^{\perp}$, and $\ell$ and the zero locus of $g_{3}$ intersect in a point. Let $g_{1}=l \tilde{g}_{1}$ and $g_{2}=l \tilde{g}_{2}$, where $l$ is the linear factor corresponding to $\ell$. Then a subscheme of length 3 of $\Gamma$ is contained in the zero-locus of $\tilde{g}_{1}$ and $\tilde{g}_{2}$. This forces $\tilde{g}_{1}$ and $\tilde{g}_{2}$ to have a common component $\tilde{\ell}$, because otherwise their zero locus (being the intersection of two $(1,1)$-curves) would have length at most 2 . Then $\tilde{\ell}$ is either a $(1,0)$ or a $(0,1)$-line. In the first case, we can repeat the previous argument and apply $\delta_{1,2}$ to $\Gamma$, obtaining a contradiction; in the second case we use $\delta_{2,1}$. The case with $I_{\Gamma,(1,2)}$ is analogous.

Corollary 3.12. For a general bihomogeneous form $f$ of bidegree $(2,2)$ the variety $\operatorname{VPS}_{\mathbb{P}^{1} \times \mathbb{P}^{1}}([f], 4)$ is a smooth 3 -fold.

Proof. First of all, the Hilbert scheme Hilb $\left(\mathbb{P}^{1} \times \mathbb{P}^{1}\right)$ itself is smooth (see [7]). Consider next, in $\mathrm{Hilb}_{4}\left(\mathbb{P}^{1} \times \mathbb{P}^{1}\right)$, the open subset $\mathcal{U}$ of schemes $\Gamma$ whose ideal $I_{\Gamma} \subseteq T$ 
has codimension 4 in bidegree $(2,2)$. Over $\mathcal{U}$ we consider the rank 5 vector bundle $E_{\mathcal{U}}$ with fiber over a scheme $[\Gamma]$ the dual of the space $I_{\Gamma,(2,2)} \subseteq T_{2,2}$ of $(2,2)$-forms in the ideal of $\Gamma$. The linear form

$$
\phi_{f,(2,2)}: T_{2,2} \longrightarrow \mathbb{C}
$$

defines a section on $E_{\mathcal{U}}$. If $\operatorname{VPS}_{\mathbb{P}^{1} \times \mathbb{P}^{1}}([f], 4) \subseteq \mathcal{U}$, then $\operatorname{VPS}_{\mathbb{P}^{1} \times \mathbb{P}^{1}}([f], 4)$ is the 0-locus of such section by Proposition 3.11, since in the case of surfaces all apolar schemes are in the closure of smooth apolar schemes.

Lemma 3.13. Let $f \in S_{2,2}$ be general, then $\operatorname{VPS}_{\mathbb{P}^{1} \times \mathbb{P}^{1}}([f], 4) \subseteq \mathcal{U}$.

Proof. It suffices to prove that for any $[\Gamma] \in \operatorname{VPS}_{\mathbb{P}^{1} \times \mathbb{P}^{1}}([f], 4)$, the space $I_{\Gamma,(2,2)}$ has dimension 5 , or equivalently, the image $\nu_{2,2}(\Gamma)$ spans a $\mathbb{P}^{3}$. For this, assume that $\nu_{2,2}(\Gamma)$ spans a plane $P_{\Gamma}$.

If the intersection $P_{\Gamma} \cap \nu_{2,2}\left(\mathbb{P}^{1} \times \mathbb{P}^{1}\right)$ is finite, $\Gamma$ is either curvilinear or it contains the neighborhood of a point. In the latter case, $P_{\Gamma}$ must be a tangent plane to $\nu_{2,2}\left(\mathbb{P}^{1} \times \mathbb{P}^{1}\right)$, but a tangent plane intersects $\nu_{2,2}\left(\mathbb{P}^{1} \times \mathbb{P}^{1}\right)$ only in a scheme of length 3 , so this is impossible. If $\Gamma$ is curvilinear it is contained in a smooth hyperplane section of $\nu_{2,2}(\Gamma)$, an elliptic normal curve of degree 8. But on any such curve any subscheme of length 4 spans a $\mathbb{P}^{3}$, again a contradiction.

Finally, if $P_{\Gamma} \cap \nu_{2,2}\left(\mathbb{P}^{1} \times \mathbb{P}^{1}\right)$ is infinite, it contains a curve. But the only plane curves on $\nu_{2,2}\left(\mathbb{P}^{1} \times \mathbb{P}^{1}\right)$ are conics, and they are the intersection of their span with $\nu_{2,2}\left(\mathbb{P}^{1} \times \mathbb{P}^{1}\right)$. So, in this case, $\Gamma$ is contained in a $(0,1)$-curve or a $(1,0)$-curve. If $\Gamma$ is apolar to $f$, this is impossible, so the lemma follows.

Taking all $\phi_{f,(2,2)}$ for $f \in S_{2,2}$ gives a linear space of sections of $E_{\mathcal{U}}$ without basepoints on $\mathcal{U}$, so for a general $f$ the 0 -locus $\operatorname{VPS}_{\mathbb{P}^{1} \times \mathbb{P}^{1}}([f], 4)$ is smooth. This proves Corollary 3.12 .

Theorem 1.4(A) is now equivalent to the following:

Theorem 3.14. For a general bihomogeneous form $f$ of bidegree $(2,2)$, the variety $\operatorname{VPS}_{\mathbb{P}^{1} \times \mathbb{P}^{1}}([f], 4)$ is isomorphic to the graph of the birational automorphism on a smooth quadric threefold $Q$ given by the linear system of quadrics through a rational normal quartic curve in $Q$.

Proof. We first show that the following rational map is an injective morphism:

$$
\begin{aligned}
\Xi: \operatorname{VPS}_{\mathbb{P}^{1} \times \mathbb{P}^{1}}([f], 4) & -\rightarrow G\left(2, f_{2,1}^{\perp}\right) \times G\left(2, f_{1,2}^{\perp}\right) \\
{[\Gamma] } & \mapsto \quad\left(I_{\Gamma,(2,1)}, I_{\Gamma,(1,2)}\right)
\end{aligned}
$$


From Proposition 3.11 all schemes $[\Gamma] \in \operatorname{VPS}_{\mathbb{P}^{1} \times \mathbb{P}^{1}}([f], 4)$ are apolar to $f$, so $I_{\Gamma} \subseteq f^{\perp}$. By Remark 3.6, both images of $\Gamma$ under $\delta_{2,1}$ and $\delta_{1,2}$ lie exactly on one line, so

$$
\operatorname{dim} I_{\Gamma,(1,2)}=\operatorname{dim} I_{\Gamma,(2,1)}=2 .
$$

Hence $\Xi$ is a morphism.

We now show the injectivity of $\Xi$. From Lemma 3.7 and the fact that $\Gamma$ is apolar to $f$ we have that the only points where $\Xi^{-1}$ is possibly not defined are the images of schemes $\Gamma$ that contain a subscheme of length 2 on a (1,0)-line, and a subscheme of length 2 on a $(0,1)$-line. If these two subschemes of $\Gamma$ do not intersect, then the union of the two lines is defined by a $(1,1)$-form that must be apolar to $f$, contradicting Lemma 3.1. If the two subschemes intersect, the scheme $\Gamma$ is mapped to a line in both $Z_{2,1}$ and $Z_{1,2}$. In this case, $\Gamma$ has a subscheme of length 3 contained in the union of a $(0,1)$-line and a $(1,0)$-line and a residual point that lies in the double curve and thus is mapped to the singular curve in both $Z_{2,1}$ and $Z_{1,2}$.

Assume that $\Gamma$ and $\Gamma^{\prime}$ are two apolar schemes of length 4 and that $\Xi(\Gamma)=\Xi\left(\Gamma^{\prime}\right)$. Then both $\Gamma$ and $\Gamma^{\prime}$ have a subscheme of length 3 contained in a pair of lines $L \cup L^{\prime}$ that together form a $(1,1)$-curve, and they each have a residual point that is mapped to a singular point in both $Z_{2,1}$ and $Z_{1,2}$. Then for each line $L$ and $L^{\prime}$ the subschemes of $\Gamma$ and $\Gamma^{\prime}$ residual to the line must coincide. But both schemes must also contain the point of intersection of $L$ and $L^{\prime}$, so the two schemes coincide. Hence $\Xi$ is injective.

Since $\operatorname{VPS}_{\mathbb{P}^{1} \times \mathbb{P}^{1}}([f], 4)$ is smooth, to complete the proof it suffices to identify the image of $\Xi$ and show that it is smooth.

Now, the collection of lines $D_{2,1} \subseteq X_{2,1}$ as defined in (7) is a smooth rational quartic curve. It is normal; otherwise, it would span a $\mathbb{P}^{3}$ and therefore be contained in a special linear complex, i.e. all lines in $Z_{2,1}$ would intersect some fixed line, which is ruled out by Corollary 3.3 above. In the planes spanned by two intersecting lines in $Z_{2,1}$ the pencil of lines through the intersection point is a line in $X_{2,1}$. For each double point on $Z_{2,1}$ we obtain such a line, so they form a surface scroll $V_{2,1} \subseteq X_{2,1}$. By construction, $D_{2,1}$ is contained in this scroll and intersects the general line in the scroll in two points. So $V_{2,1}$ is also contained in the secant variety of $D_{2,1}$, a cubic hypersurface $S D_{2,1}$. Therefore, the scroll has degree at most 6 and is contained in the complete intersection $S D_{2,1} \cap X_{2,1}$.

To see that $V_{2,1}=S D_{2,1} \cap X_{2,1}$ we compute its degree. This is computed from the bidegree $\left(d_{1}, d_{2}\right)$ in the Grassmannian. Notice that $V_{2,1}$ parametrizes the lines in $X_{2,1}$ that pass through a singular point in $Z_{2,1}$. Moreover, lines $\ell$ in $\mathbb{P}\left(\left(f_{2,1}^{\perp}\right)^{*}\right)$ that pass through a singular point $Q$ in $Z_{2,1}$ such that $[\ell] \in X_{2,1}$, all lie in the plane spanned by the two lines contained in $Z_{2,1}$ passing through $Q$. The number $d_{1}$ counts the number of lines in a general plane that belong to $V_{2,1}$. A general plane 
$P$ contains three singular points of $Z_{2,1}$. For each of them, there is one line contained in both $Z_{2,1}$ and $P$ passing through it, so $d_{1}=3$. The number of lines through a general point that belong to $V_{2,1}$ is $d_{2}$. A general point lies in three planes that intersect $Z_{2,1}$ in a conic section, hence also in two lines, so $d_{2}=3$. We conclude that $V_{2,1}$ has degree 6 , and so $V_{2,1}$ is a complete intersection.

Consider now a Veronese surface $\mathcal{V} \subseteq G\left(2, f_{2,1}^{\perp}\right)$ that contains $D_{2,1}$. The Cremona transformation on $\mathbb{P}^{5}$ defined by the quadrics in the ideal of $\mathcal{V}$ contracts the secant variety of $\mathcal{V}$ to a Veronese surface $\mathcal{V}^{\prime}$, while the strict transform of $\mathcal{V}$ is mapped to the secant variety of $\mathcal{V}^{\prime}$. The Cremona transformation restricts to a birational map

$$
\gamma_{2,1}: X_{2,1}-\rightarrow X^{\prime} \subseteq \mathbb{P}^{4}
$$

where $X^{\prime} \subseteq \mathbb{P}^{4}$ is a smooth quadric 3 -fold. In fact, the restriction is defined by the quadrics in the ideal of $D_{2,1}$ in $X_{2,1}$. This space of quadrics is 5-dimensional, and the image is a hyperplane section $X^{\prime}$ of the Plücker quadric, defined by the quadratic relation between the quadrics in the ideal of $D_{2,1}$ as a curve in $\mathbb{P}^{4}$.

Consider the closure of the graph $\mathcal{Y} \subseteq X_{2,1} \times X^{\prime}$ of the rational map $\gamma_{2,1}$. The strict transform of $D_{2,1}$ in $\mathcal{Y}$ is mapped to a scroll $T^{\prime}$ in $X^{\prime}$, the intersection of the secant variety of the Veronese surface $\mathcal{V}^{\prime}$ with the quadric threefold $X^{\prime}$. The strict transform in $\mathcal{Y}$ of $V_{2,1}$ is mapped to a rational normal quartic curve $C^{\prime}$.

We now compare the map $\gamma_{2,1}$ with the natural birational map $\rho$ sending $[\ell]$ to $\Phi_{1,2}\left(\Phi_{2,1}^{-1}([\ell])\right)$

$$
\rho: X_{2,1}-\rightarrow X_{1,2} \text {. }
$$

Since $\Phi_{2,1}$ is bijective outside the preimage of the curve $D_{2,1}$, the rational map $\rho$ is defined outside $D_{2,1}$. On the other hand, $\rho$ is not defined anywhere on $D_{2,1}$. The Picard group of $X_{2,1}$ is generated by the hyperplane bundle, so the map $\rho$ must be defined by a 5 -dimensional space of sections in $\mathrm{H}^{0}\left(\mathcal{I}_{D_{2,1}}(d)\right)$ for some $d$, where $\mathcal{I}_{D_{2,1}}$ is the sheaf of ideals of $D_{2,1}$ on the quadric 3 -fold $X_{2,1}$. To find the degree $d$ we consider a general curve $C$ defined by a section in $f_{2,1}^{\perp}$. On the surface $Z_{2,1} \subseteq \mathbb{P}^{3}$ the curve $C$ is mapped to a plane quartic curve with a linear pencil of lines that cut the curve in the image of schemes of length 4 that are apolar to $f$. This pencil forms a line in $X_{2,1}$ that does not intersect $D_{2,1}$. Now, the image $\bar{C}$ of the curve $C$ on $Z_{1,2}$ has degree 5 . The pencil of apolar schemes of length 4 on $C$ is mapped to schemes that are collinear also in $Z_{1,2}$, so it defines on $\bar{C}$ a pencil of 4 -secant lines. Assuming $C$ is smooth, any two of these 4 -secant lines are disjoint; otherwise, $\bar{C}$ would have a plane section of length 7 or 8 , impossible. Therefore, the pencil of 4 -secant lines are the lines of one family of lines in a smooth quadric surface. This means that the image of this pencil of lines in $X_{1,2}$ is a conic, and hence the degree $d$ is 2 . Since

$$
\operatorname{dim} \mathrm{H}^{0}\left(\mathcal{I}_{D_{2,1}}(2)\right)=5
$$


we may conclude that the map $\rho$ coincides with $\gamma_{2,1}$. Clearly $\gamma_{1,2}$ is the inverse of $\gamma_{2,1}$, and the graph $\mathcal{Y}$ of $\rho$ is the blowup of $X_{2,1}$ along the smooth curve $D_{2,1}$, so $\mathcal{Y}$ is smooth. There is a map from the graph of $\gamma_{1,2}$ to $\operatorname{VPS}_{\mathbb{P}^{1} \times \mathbb{P}^{1}}([f], 4)$ which sends a graph point to the ideal generated by the two pencils, one in each Grassmannian. Therefore, the graph $\mathcal{Y}$ is identified with $\Xi\left(\operatorname{VPS}_{\mathbb{P}^{1} \times \mathbb{P}^{1}}([f], 4)\right)$. The graph is smooth, so $\operatorname{VPS}_{\mathbb{P}^{1} \times \mathbb{P}^{1}}([f], 4)$ and the graph $\mathcal{Y}$ are isomorphic.

\section{Bihomogeneous forms of bidegree $(3,3)$}

Let $f$ be a bihomogeneous form in $S$ of bidegree $(3,3)$. The Segre-Veronese embedding (3) is in this case $\nu_{3,3}: \mathbb{P}^{1} \times \mathbb{P}^{1} \hookrightarrow \mathbb{P}^{15}$. By $(4)$, we have $\operatorname{rank}(f)=6$ and $\operatorname{dim} \operatorname{VPS}_{\mathbb{P}^{1} \times \mathbb{P}^{1}}([f], 6)=2$.

We identify $\mathbb{P}\left(S_{3,3}\right)$ with a linear subspace

$$
\mathbb{P}^{15} \subseteq \mathbb{P}\left(\mathbb{C}\left[z_{0}, z_{1}, z_{2}, z_{3}\right]_{3}\right)=\mathbb{P}^{19}
$$

where $z_{0}=x_{0} y_{0}, z_{1}=x_{0} y_{1}, z_{2}=x_{1} y_{0}, z_{3}=x_{1} y_{1}$. So we can see $[f] \in \mathbb{P}^{15}$ as a cubic form $[F] \in \mathbb{P}^{19}$ in 4 variables.

Remark 4.1. Given $f \in S_{3,3}$ we can associate to it two orthogonal ideals: first of all we have the orthogonal $f^{\perp} \subseteq \mathbb{C}\left[t_{0}, t_{1}\right]\left[u_{0}, u_{1}\right]$ that we introduced and used in the previous sections; moreover, once we interpret $f$ as a cubic form $F$, we have also $F^{\perp} \subseteq \mathbb{C}\left[v_{0}, v_{1}, v_{2}, v_{3}\right]$, a homogeneous ideal in a polynomial ring in 4 variables, that act on $\mathbb{C}\left[z_{0}, z_{1}, z_{2}, z_{3}\right]$ by differentiation, namely $v_{i}(f)=\partial / \partial z_{j}(f)$ for $f \in \mathbb{C}\left[z_{0}, z_{1}, z_{2}, z_{3}\right]$.

Lemma 4.2. For a general bihomogeneous form $f \in S_{3,3}$, the orthogonal $f^{\perp}$ is generated by 5 bihomogeneous forms of bidegree $(2,2)$ in $T$, together with $f_{3,1}^{\perp}, f_{1,3}^{\perp}$, $f_{4,0}^{\perp}$ and $f_{0,4}^{\perp}$.

Proof. Let us consider the maps $\phi_{f,(a, b)}$ as we did in Section 3. The kernels of these maps are the bihomogeneous components of the orthogonal ideal of $f$. Since $f$ is a general form, we may assume that the maps $\phi_{f,(a, b)}$ have maximal rank, i.e. are either injective or surjective. Thus, we may assume they are injective when

$$
(a, b) \in\{(0,0),(0,1),(1,0),(1,2),(2,1),(0,3),(3,0)\} .
$$

Then, since $\operatorname{dim} T_{2,2}=9$ and $\operatorname{dim} S_{1,1}=4$, the map $\phi_{f,(2,2)}$ is surjective and $\operatorname{dim} f_{2,2}^{\perp}=5$. Similarly, we can see that the dimension of $f_{3,1}^{\perp}$ and $f_{1,3}^{\perp}$ is also 5 , and that

$$
\operatorname{dim} f_{2,3}^{\perp}=\operatorname{dim} f_{3,2}^{\perp}=10 \quad \text { and } \quad \operatorname{dim} f_{3,3}^{\perp}=15 .
$$


By an analogous procedure to that in the proof of Lemma 3.1, it follows that $f_{2,3}^{\perp}=$ $T_{0,1} \cdot f_{2,2}^{\perp}$ and $f_{3,2}^{\perp}=T_{1,0} \cdot f_{2,2}^{\perp}$, and that $f^{\perp}$ is generated by $f_{a, b}^{\perp}$ for $a, b \leq 3$ together with $T_{4,0}=f_{4,0}^{\perp}$ and $T_{0,4}=f_{0,4}^{\perp}$.

We are left to prove that $f_{3,3}^{\perp}$ is generated by $f_{2,2}^{\perp}, f_{3,1}^{\perp}$, and $f_{1,3}^{\perp}$. If not, then in particular the multiplication map $f_{1,3}^{\perp} \otimes T_{2,0} \longrightarrow f_{3,3}^{\perp}$, is not onto. But then there is a relation $g q-g^{\prime} q^{\prime}=0$, where say $g, g^{\prime} \in f_{1,3}^{\perp}$, while $q, q^{\prime} \in T_{2,0}$. By unique factorization, $q$ and $q^{\prime}$ must have a common factor, so $g l=g^{\prime} l^{\prime}$ for some $l, l^{\prime} \in T_{1,0}$. By assumption, $g, g^{\prime}$ are independent, so $l, l^{\prime}$ generate $T_{1,0}$ and $g=g_{0} l^{\prime}$ and $g^{\prime}=g_{0} l$. This is possible only if $g_{0} \in f_{0,3}^{\perp}$, against our assumption.

Let $f$ be a general bihomogeneous form of bidegree $(3,3)$ and let $F \in \mathbb{C}\left[z_{0}, z_{1}\right.$, $z_{2}, z_{3}$ ] be the cubic associated to $f$. If $F$ is not a cone, the orthogonal $F^{\perp}$ is generated by 6 quadrics. By Sylvester's Pentahedral Theorem (see [23] and for example [17, Theorem 3.9] and [14, Example 12.4.2.3]) the powersum variety $\operatorname{VSP}(F, 5)=$ $\operatorname{VPS}_{\mathbb{P}^{3}}([F], 5)$ is just a point corresponding to a scheme $\Gamma_{0} \subseteq \mathbb{P}^{3}$ given by a set of 5 points. The ideal of $\Gamma_{0}$ is generated by 5 quadrics, so a general quadric apolar to $F$ does not intersect $\Gamma_{0}$. In fact, we may assume that $\nu_{1,1}\left(\mathbb{P}^{1} \times \mathbb{P}^{1}\right)$ in $\mathbb{P}^{3}$ is defined by a general quadric polynomial orthogonal to $F$, and hence

$$
\Gamma_{0} \cap \nu_{1,1}\left(\mathbb{P}^{1} \times \mathbb{P}^{1}\right)=\varnothing
$$

We consider the closure $H_{3 t+1}\left(\Gamma_{0}\right)$ in the Hilbert scheme of twisted cubic curves, of the set of curves that contain $\Gamma_{0}$. A result of Kapranov shows that it is a smooth surface.

Proposition 4.3. ([13, Theorem 4.3.3]) $H_{3 t+1}\left(\Gamma_{0}\right)$ is isomorphic to a smooth Del Pezzo surface of degree 5, i.e. isomorphic to the blowup of $\mathbb{P}^{2}$ in 4 points.

Lemma 4.4. Let $f$ be a general bihomogeneous form of bidegree $(3,3)$, and let $\Gamma_{0}$ be the unique set of 5 points in $\mathbb{P}^{3}$ that is apolar to the cubic form $F$ corresponding to $f$. Then for every smooth apolar $[\Gamma] \in \operatorname{VPS}_{\mathbb{P}^{1} \times \mathbb{P}^{1}}([f], 6)$, there exists a (possibly reducible) twisted cubic curve $C_{\Gamma}$ passing through $\Gamma_{0}$ and $\Gamma$, in particular $\left[C_{\Gamma}\right] \in$ $H_{3 t+1}\left(\Gamma_{0}\right)$.

Proof. Consider 6 general points on $\nu_{1,1}\left(\mathbb{P}^{1} \times \mathbb{P}^{1}\right) \subseteq \mathbb{P}^{3}$. They are the intersection of $\nu_{1,1}\left(\mathbb{P}^{1} \times \mathbb{P}^{1}\right)$ with a twisted cubic curve. This is a particular case of a classical result often called Castelnuovo's lemma: through $n+3$ points in $\mathbb{P}^{n}$, no $n$ of which lie in a $\mathbb{P}^{n-2}$, there is a unique reduced and connected curve of degree $n$ and arithmetic genus 0 .

Therefore, if $[\Gamma] \in \operatorname{VPS}_{\mathbb{P}^{1} \times \mathbb{P}^{1}}([f], 6)$ is an apolar scheme constituted of 6 general points, then $\Gamma \subseteq C_{\Gamma} \subseteq \mathbb{P}^{3}$, where $C_{\Gamma}$ is a twisted cubic. One can also show that 
$\Gamma_{0} \subseteq C_{\Gamma}$. In fact, by the apolarity lemma, it follows that $I_{\Gamma} \subseteq F^{\perp}$, and since $I_{C_{\Gamma}} \subseteq I_{\Gamma}$ we get $I_{C_{\Gamma}} \subseteq F^{\perp}$. Under the 3 -uple Veronese embedding $C_{\Gamma}$ becomes a rational curve of degree 9 , and since $C_{\Gamma}$ is apolar to $F$, the point $[F]$ lies in the span of this degree 9 curve. Therefore, $F$ can be interpreted as a general binary form of degree 9, and by Lemma 1.5 such a binary form has rank 5 , so $[F]$ lies on the span of 5 points belonging to the degree 9 curve. On the other hand, the only scheme of 5 points apolar to $F$ is $\Gamma_{0}$; therefore, those 5 points are nothing but the image of $\Gamma_{0}$ under the 3-uple Veronese embedding, which implies that $C_{\Gamma}$ passes through $\Gamma_{0}$.

We consider now the other kinds of smooth apolar schemes in $\operatorname{VPS}_{\mathbb{P}^{1} \times \mathbb{P}^{1}}([f], 6)$. If no plane passes through 4 of the points of $\Gamma$, then we are in the general situation and the previous argument shows that we have a unique (smooth) twisted cubic through $\Gamma$ and $\Gamma_{0}$. Suppose that exactly 4 points of $\Gamma$ lie on a plane, and no three of them are on a line. Then there is a pencil of conics passing through those planar points, and a line $\ell$ through the remaining two points; thus there exists a unique conic $C$ in this pencil meeting $\ell$. We prove that $\Gamma_{0}$ is contained in $C \cup \ell$, which hence is an element in $H_{3 t+1}\left(\Gamma_{0}\right)$ (corresponding in the Del Pezzo surface to a point lying on one of the 10 lines of the surface). Under the 3 -uple Veronese embedding, the line $\ell$ is mapped to a twisted cubic $D_{1}$, and the conic $C$ is mapped to a rational sextic $D_{2}$. By construction, the point $[F]$ lies on the span of $D_{1} \cup D_{2}$. We denote by $Q$ the point of intersection between $D_{1}$ and $D_{2}$. We use the same technique as in the proof of Lemma 3.8 to construct a scheme of length 5 apolar to $F$. Let $E_{1}=\operatorname{span} D_{1}$ and $E_{2}=\operatorname{span} D_{2}$, then $E_{1} \cong \mathbb{P}^{3}$ and $E_{2} \cong \mathbb{P}^{6}$. After projection from the point $[F]$ into $\mathbb{P}^{8}$ the two linear spaces $E_{1}$ and $E_{2}$ will intersect in a line, so there is a unique plane $P$ containing the line $\overline{[F] Q}$ and intersecting $E_{1}$ in a line $\ell_{E_{1}}$ and $E_{2}$ in a line $\ell_{E_{2}}$. The variety of 3 -secant planes to $D_{2}$ is a quartic hypersurface in $E_{2}$, and a general line meeting $D_{2}$ intersects it in a unique further point. In particular $\ell_{E_{2}}$ intersects $D_{2}$ in $Q$ and the variety of 3 -secant planes in a further point $T$. Therefore, we may assume that there are three points $p_{1}, p_{2}$ and $p_{3}$ in $D_{2}$ whose span contains $T$. Consider now the line $\overline{[F] T}$ : since it is contained in $P$, it meets $\ell_{E_{1}}$ in one point $R$. A general point in $E_{1}$ lies in a unique secant to $D_{1}$, so we obtain two points $p_{4}, p_{5}$ in $D_{2}$ whose span contains $R$. In this way $[F] \in \operatorname{span}\left(\left\{p_{1}, \ldots, p_{5}\right\}\right)$. As above $\left\{p_{1}, \ldots, p_{5}\right\}=\Gamma_{0}$ under the 3 -uple Veronese embedding, so the lemma follows.

Eventually, we rule out all the cases that are left. Suppose that 3 of the 6 points of $\Gamma$ are collinear on a line $\ell$; then those 3 collinear points may be replaced in $\Gamma$ by a scheme of length 2 , so that $f$ is apolar to a scheme of length 5 on $\mathbb{P}^{1} \times \mathbb{P}^{1}$. If 5 of the 6 points lie in a plane then, the five coplanar points may be replaced in $\Gamma$ by a scheme of length 4 , so that $f$ is apolar to a scheme of length 5 on $\mathbb{P}^{1} \times \mathbb{P}^{1}$. In both cases this is against the generality assumption of $f$. 
We now reformulate and prove Theorem 1.4(B).

Theorem 4.5. For a general bihomogeneous form $f$ of bidegree $(3,3)$ the variety $\operatorname{VPS}_{\mathbb{P}^{1} \times \mathbb{P}^{1}}([f], 6)$ is isomorphic to a smooth Del Pezzo surface of degree 5 .

Proof. Let $\Gamma_{0}$ be the set of 5 points apolar to the cubic form $F$ associated to $f$ as in Lemma 4.4. Let $H_{3 t+1}\left(\Gamma_{0}\right)$ be the Hilbert scheme of twisted cubic curves through $\Gamma_{0}$.

If $[C] \in H_{3 t+1}\left(\Gamma_{0}\right)$, then $C$ is a cubic curve through $\Gamma_{0}$, that is apolar to $F$. Moreover, $\nu_{1,1}\left(\mathbb{P}^{1} \times \mathbb{P}^{1}\right) \cap C$ is a scheme of length 6 . In fact, every component of $C$ contains some subset of $\Gamma_{0}$ and therefore intersects $\nu_{1,1}\left(\mathbb{P}^{1} \times \mathbb{P}^{1}\right)$ properly. Thus, we get a morphism

$$
\psi: H_{3 t+1}\left(\Gamma_{0}\right) \longrightarrow \operatorname{VPS}_{\mathbb{P}^{1} \times \mathbb{P}^{1}}([f], 6)
$$

This morphism is injective, because otherwise there would be two cubic curves $C$ and $C^{\prime}$ that pass through $\Gamma_{0}$ and have a common intersection with $\nu_{1,1}\left(\mathbb{P}^{1} \times \mathbb{P}^{1}\right)$. Since $\Gamma_{0}$ has no common point with $\nu_{1,1}\left(\mathbb{P}^{1} \times \mathbb{P}^{1}\right)$ this is impossible by Castelnuovo's lemma. To show that the morphism $\psi$ is surjective, we first note that both $H_{3 t+1}\left(\Gamma_{0}\right)$ and the variety $\operatorname{VPS}_{\mathbb{P}^{1} \times \mathbb{P}^{1}}([f], 6)$ are surfaces, so it suffices to show that $\psi$ is onto the set of smooth schemes in $\operatorname{VPS}_{\mathbb{P}^{1} \times \mathbb{P}^{1}}([f], 6)$. This is precisely the content of Lemma 4.4.

It remains to show that the bijective morphism $\psi$ is an isomorphism.

Lemma 4.6. If $f$ is a general $(3,3)$-form and $[\Gamma] \in \operatorname{VPS}_{\mathbb{P}^{1} \times \mathbb{P}^{1}}([f], 6)$ then $\Gamma$ is apolar to $f$.

Proof. The ideal of each curve $C$ in $H_{3 t+1}\left(\Gamma_{0}\right)$ is contained in the ideal of $\Gamma_{0}$ and is therefore apolar to the cubic form $F$ associated to $f$. The scheme of intersection $\nu_{1,1}\left(\mathbb{P}^{1} \times \mathbb{P}^{1}\right) \cap C$ is therefore apolar to $f$. This intersection has length 6 and belongs to the closure of the smooth apolar schemes in $\operatorname{VPS}_{\mathbb{P}^{1} \times \mathbb{P}^{1}}([f], 6)$. Since $\psi$ is a surjective morphism, the lemma follows.

To show that $\psi$ is an isomorphism, we show that $\operatorname{VPS}_{\mathbb{P}^{1} \times \mathbb{P}^{1}}([f], 6)$ is smooth. First of all, the Hilbert scheme $\operatorname{Hilb}_{6}\left(\mathbb{P}^{1} \times \mathbb{P}^{1}\right)$ itself is smooth (see [7]). Consider next, in $\operatorname{Hilb}_{6}\left(\mathbb{P}^{1} \times \mathbb{P}^{1}\right)$, the open subset $\mathcal{U}$ of schemes $[\Gamma]$ that lie on a unique curve in the Hilbert scheme of twisted cubic curves in $\mathbb{P}^{3}$, and whose ideal on $\mathbb{P}^{1} \times \mathbb{P}^{1}$ has $\operatorname{dim} I_{\Gamma,(3,3)}=10$, or equivalently, such that the span of $\nu_{3,3}(\Gamma)$ is a $\mathbb{P}^{5}$. Over $\mathcal{U}$ we consider the rank 10 vector bundle $E_{\mathcal{U}}$ whose fiber over a scheme $[\Gamma]$ is the dual of the space $I_{\Gamma,(3,3)} \subseteq T_{3,3}$ of $(3,3)$-forms in the ideal of $\Gamma$. The linear form $\phi_{f,(3,3)}$ : $T_{3,3} \longrightarrow \mathbb{C}$ defines a section on $E_{\mathcal{U}}$. If $\operatorname{VPS}_{\mathbb{P}^{1} \times \mathbb{P}^{1}}([f], 6) \subseteq \mathcal{U}$, then $\operatorname{VPS}_{\mathbb{P}^{1} \times \mathbb{P}^{1}}([f], 6)$ is the 0-locus of this section, by Lemma 4.6.

Lemma 4.7. If $f \in S_{3,3}$ is general, then $\operatorname{VPS}_{\mathbb{P}^{1} \times \mathbb{P}^{1}}([f], 6) \subseteq \mathcal{U}$. 
Proof. It suffices to prove that for any $[\Gamma] \in \operatorname{VPS}_{\mathbb{P}^{1} \times \mathbb{P}^{1}}([f], 6)$ the span of $\nu_{3,3}(\Gamma)$ is a $\mathbb{P}^{5}$. For a general $f$, consider the 5 points $\Gamma_{0}$ apolar to the cubic form $F$ on $\mathbb{P}^{3}$ associated to $f$. We may assume that every line through a pair of points of $\Gamma_{0}$ intersects $\nu_{1,1}\left(\mathbb{P}^{1} \times \mathbb{P}^{1}\right)$ transversally. Therefore, the intersection of any cubic curve in $H_{3 t+1}\left(\Gamma_{0}\right)$ with $\nu_{1,1}\left(\mathbb{P}^{1} \times \mathbb{P}^{1}\right)$ is curvilinear. If the cubic curve has a component of degree $d$, then the intersection with $\nu_{1,1}\left(\mathbb{P}^{1} \times \mathbb{P}^{1}\right)$ has degree $2 d$. On the 3 -uple embedding of this curve, any such curvilinear scheme spans a $\mathbb{P}^{5}$.

Taking all $\phi_{f,(3,3)}$ for $f \in S_{3,3}$ gives a linear space of sections of $E_{\mathcal{U}}$ without basepoints on $\mathcal{U}$, so for a general $f$ the 0 -locus $\operatorname{VPS}_{\mathbb{P}^{1} \times \mathbb{P}^{1}}([f], 6)$ is smooth. Since $\psi$ is a bijective map between smooth surfaces, it is an isomorphism.

\section{Cubic forms on a cubic surface scroll}

Let $\Sigma$ be a cubic scroll in $\mathbb{P}^{4}$. The Picard group $\operatorname{Pic}(\Sigma)$ is free of rank 2 generated by the class of curves $E$ and $F$, where $E^{2}=-1, E \cdot F=1$ and $F^{2}=0$, see for instance [1, Proposition IV.1] or [8, Chap. 5, Lemma 2]. The linear system $|E+F|$ defines a morphism $\pi: \Sigma \longrightarrow \mathbb{P}^{2}$, which is the blowup of a point $p_{E} \in \mathbb{P}^{2}$ with exceptional divisor $\pi^{-1}\left(p_{E}\right)=E$. The Cox ring of $\Sigma$ is isomorphic to a bihomogeneous polynomial ring $T=\mathbb{C}\left[t_{0}, t_{1}, u_{0}, u_{1}\right]$ such that

$$
\begin{aligned}
T_{E} & =\mathrm{H}^{0}\left(\Sigma, \mathcal{O}_{\Sigma}(E)\right)=\left\langle t_{0}\right\rangle, \\
T_{F} & =\mathrm{H}^{0}\left(\Sigma, \mathcal{O}_{\Sigma}(F)\right)=\left\langle u_{0}, u_{1}\right\rangle, \\
T_{E+F} & =\mathrm{H}^{0}\left(\Sigma, \mathcal{O}_{\Sigma}(E+F)\right)=\left\langle t_{0} u_{0}, t_{0} u_{1}, t_{1}\right\rangle .
\end{aligned}
$$

Let $S=\mathbb{C}\left[x_{0}, x_{1}, y_{0}, y_{1}\right]$ with $t_{0}, t_{1}$ dual to $x_{0}, x_{1}$ and $u_{0}, u_{1}$ dual to $y_{0}, y_{1}$, generating an action of $T$ on $S$ by differentiation, that defines the apolarity of the introduction in coordinates. In fact, we may then interpret $\Sigma \subseteq \mathbb{P}\left(S_{E+2 F}\right)$ as a set of forms:

$$
\begin{aligned}
& \Sigma=\left\{\left[a_{0} x_{0} l\left(y_{0}, y_{1}\right)+a_{1} x_{1} l\left(y_{0}, y_{1}\right)^{2}\right] \in \mathbb{P}\left(S_{E+2 F}\right):\right. \\
& \left.\quad a_{0}, a_{1} \in \mathbb{C}, l\left(y_{0}, y_{1}\right) \in\left\langle y_{0}, y_{1}\right\rangle\right\} .
\end{aligned}
$$

Let $f \in S_{3 E+6 F} \subseteq \operatorname{Sym}^{3} S_{E+2 F}$. Thus $f$ may be interpreted as a cubic form $G$ on $\mathbb{P}^{4}$ restricted to $\Sigma$. According to the definition of variety of apolar schemes of Section 1 , we have

$$
\operatorname{VPS}_{\Sigma}([f], 8)=\overline{\left\{[\Gamma] \in \operatorname{Hilb}_{8}(\Sigma):[f] \in \operatorname{span}\left(\nu_{3 E+6 F}(\Gamma)\right), \Gamma \text { smooth }\right\}},
$$

where $\nu_{3 E+6 F}$ is the morphism associated to the divisor $3 E+6 F$.

The following theorem is equivalent to Theorem 1.4(C). 
Theorem 5.1. For a general $f \in S_{3 E+6 F}$, the variety $\operatorname{VPS}_{\Sigma}([f], 8)$ is isomorphic to $\mathbb{P}^{2}$ blown up in 8 points.

Proof. Recall that we may interpret $[f]$ as a point $[G]$ in the linear span inside $\mathbb{P}^{34}=\mathbb{P}\left(\mathbb{C}\left[z_{0}, z_{1}, z_{2}, z_{3}, z_{4}\right]_{3}\right)$ of the 3 -uple embedding of $\Sigma$. We may clearly interpret $G$ as a general cubic form in $\mathbb{P}^{4}$. Therefore $G$, and hence $f$, is not apolar to any rational quartic curve. In particular, we may assume that $I_{f, 2 E+2 F}=I_{f, E+3 F}=0$. Furthermore, we may assume that

$$
\phi_{f, 2 E+3 F}: T_{2 E+3 F} \longrightarrow S_{E+3 F}
$$

has maximal rank, so $I_{f, 2 E+3 F}=\operatorname{ker} \phi_{f, 2 E+3 F}$ is 2-dimensional, i.e. defines a pencil of curves $K \subseteq|2 E+3 F|$. Notice that, by the apolarity lemma 1.3, every curve in $K$ is apolar to $f$.

Lemma 5.2. For a general $f \in S_{3 E+6 F}$, the singular curves in $K$ are irreducible nodal curves and the basepoints $\Gamma_{0}$ of $K$ are 8 general points in $\Sigma$.

Proof. Let $\Gamma_{0} \subseteq \Sigma$ be 8 general points. In degree $2 E+3 F$, the ideal of $\Gamma_{0}$ is 2-dimensional. Furthermore, the set of forms $f^{\prime} \in S_{3 E+6 F}$ for which $\Gamma_{0}$ is apolar is the 7-dimensional subspace in $S_{3 E+6 F}$ orthogonal to $I_{\Gamma_{0}, 3 E+6 F} \subseteq T_{3 E+6 F}$. These $f^{\prime}$ are precisely the forms that are apolar to every curve in $K$. Now, $\mathbb{P}\left(S_{3 E+6 F}\right)$ has dimension 21 , while the set of pencils in $\mathbb{P}\left(T_{2 E+3 F}\right)$ has dimension 14, so the general pencil is apolar to some form $f$ and the lemma follows.

Any scheme $[\Gamma]$ in $\operatorname{VPS}_{\Sigma}([f], 8)$ has length 8 , so it lies on a curve in $|2 E+3 F|$. Therefore, if $\Gamma$ is apolar, it lies on a curve in $K$. Now the base scheme $\Gamma_{0}$ of $K$ has length 8 , so this scheme is the only one of length 8 that lies on all curves in $K$. The other schemes $\Gamma \subseteq \Sigma$ of length 8 that are apolar to $f$ lie each on a unique curve $C \in K$.

Let $C \in K$. Then $C$ is apolar to $f$, so we may consider the variety

$$
\operatorname{VPS}_{C}([f], 8) \subseteq \operatorname{VPS}_{\Sigma}([f], 8)
$$

Let $[\Gamma] \in \operatorname{VPS}_{C}([f], 8)$. Then $\Gamma \subseteq C$ is a subset of the intersection of $C$ with a curve $C^{\prime}$ in $|3 E+3 F|$. The residual part of the intersection $C \cap C^{\prime}$ is a unique point on $C$ that we denote by $p_{\Gamma}$. We thus get a map for every $C \in K$ :

$$
\psi_{C}: \operatorname{VPS}_{C}([f], 8) \longrightarrow C \text { and }[\Gamma] \longmapsto p_{\Gamma} .
$$

The map $\psi_{C}$ is defined also on $\Gamma_{0}$ since any curve $C^{\prime}+E$, with $C^{\prime} \in K$, lies in $|3 E+3 F|$ and intersect $C$ in $\Gamma_{0}$ and in the residual point $E \cap C$. 
Composing $\psi_{C}$ with the blowup map $\pi$, we get a morphism

$$
\pi \circ \psi_{C}: \operatorname{VPS}_{C}([f], 8) \longrightarrow \mathbb{P}^{2}
$$

that we want to extend to all of $\operatorname{VPS}_{\Sigma}([f], 8)$. For this, consider, in the Hilbert scheme of length 8 subschemes of $\Sigma$, the open set $\mathcal{U}$ of schemes $\Gamma$ that are contained in a unique pencil of curves $N_{\Gamma}$ in $|3 E+3 F|$. Let $\bar{\Gamma} \subseteq \Sigma$ be the baselocus of $N_{\Gamma}$. If $\bar{\Gamma}$ is finite, then it has length 9 and there is a unique point $p_{\Gamma} \in \Sigma$ residual to $\Gamma$ in $\bar{\Gamma}$. Composing with $\pi$ we get a rational map $\psi: \mathcal{U}-\rightarrow \mathbb{P}^{2}$. Clearly the restriction of $\psi$ to $\operatorname{VPS}_{C}([f], 8)$ extends to the morphism $\psi_{C}$ for every curve $C \in K$. Since $\psi_{C}\left(\Gamma_{0}\right)=\pi(E)$ for each $C$, and every other $\Gamma$ in $\operatorname{VPS}_{\Sigma}([f], 8)$ lies in a unique $C$, we see that the restriction of $\psi$ to $\operatorname{VPS}_{\Sigma}([f], 8)$ extends to a morphism

$$
\psi_{f}: \operatorname{VPS}_{\Sigma}([f], 8) \longrightarrow \mathbb{P}^{2}
$$

such that the restriction of $\psi_{f}$ to $\operatorname{VPS}_{C}([f], 8)$ coincides with $\psi_{C}$ for each $C \in K$.

We proceed to show that $\psi_{C}$ is an isomorphism for every curve $C \in K$. For this we first give a more general fact for elliptic curves, equivalent to Lemma 1.6.

Lemma 5.3. Let $C \subseteq \mathbb{P}^{2 d-2}$ be an elliptic normal curve of degree $2 d-1$, then the d-secants $\mathbb{P}^{d-1}$ 's to $C$ that pass through a general point in $\mathbb{P}^{2 d-2}$ correspond one to one to points on $C$.

Proof. Let $C^{\prime} \subseteq \mathbb{P}^{2 d-1}$ be an elliptic normal curve of degree $2 d$ embedded by a line bundle $\mathcal{L}$, then the $(d-1)$-secant variety of $C^{\prime}$ is a complete intersection of a pencil of determinantal hypersurfaces of degree $d$ : each hypersurface is defined by the minors of a matrix of linear forms (see [6, Theorem 1.3, Lemma 2.9], [21]). Furthermore, for a general line that intersects $C^{\prime}$ in a point $q$, every point outside $C^{\prime}$ lies on a unique hypersurface in the pencil, so after projecting $C^{\prime}$ from $q$ we get a curve $C$ of degree $2 d-1$. Moreover, the $d$-secants of $C$ through a general point in $\mathbb{P}^{2 d-2}$ correspond one to one to line bundles of degree $d$ on $C$, i.e. to points on $C$.

Lemma 5.4. Assume $f$ is general, so that the singular curves in $K$ are irreducible and nodal and the basepoints $\Gamma_{0}$ of $K$ are 8 points disjoint from the exceptional curve $E$. Then the morphism $\psi_{C}: \operatorname{VPS}_{C}([f], 8) \longrightarrow C$ is an isomophism for every $C \in K$, and every $[\Gamma] \in \operatorname{VPS}_{C}([f], 8)$ is apolar to $f$.

Proof. Consider the embedding $C \longrightarrow \mathbb{P}^{14} \subseteq \mathbb{P}\left(S_{3 E+6 F}\right)$ defined by the linear system $\left|(3 E+6 F)_{C}\right|$ of divisors on $C$, namely the linear system of curves $|3 E+6 F|$ restricted to $C$. It is the composition of the embedding defined by $|4 E+6 F|$ and the projection from the point $E \cap C$. We consider Weil and Cartier divisors on $C$ (if 
$C$ is smooth they of course coincide). While Weil divisors may have multiplicity one at a node $p_{C}$ of $C$, any effective Cartier divisor has multiplicity at least two at $p_{C}$. Any Weil divisor $\Gamma$ of degree 8 on $C$ is contained in a unique Cartier divisor $\bar{\Gamma}$ of degree 9 defined on $C$ by the pencil $D_{\Gamma}$ of curves in $|3 E+3 F|$ that contain $\Gamma$. The uniqueness of $D_{\Gamma}$ implies both that the map $\psi_{C}:[\Gamma] \mapsto p_{\Gamma}=\bar{\Gamma}-\Gamma$ is well-defined, and that it is injective as soon as there is a unique divisor in the linear system in $\left|(3 E+3 F)_{C}-p_{\Gamma}\right|$ that is contained in $\operatorname{VPS}_{C}([f], 8)$. Any curve $G_{\Gamma}$ in $|4 E+6 F|$ that is not a multiple of $C$ and contains the Cartier divisor $\bar{\Gamma}$, defines on $C$ a Weil divisor $\Gamma^{\prime}=G_{\Gamma} \cap C-\Gamma$ of degree 8 that contains the point $p_{\Gamma}$. Thus $\Gamma+\Gamma^{\prime}$ is a hyperplane section of $C \subseteq \mathbb{P}^{15} \subseteq \mathbb{P}\left(S_{4 E+6 F}\right)$ and we can define a pair of linear systems

$$
L_{\Gamma}:=\left|(4 E+6 F)_{C}-\Gamma^{\prime}\right| \text { and } L_{\Gamma^{\prime}}:=\left|(4 E+6 F)_{C}-\Gamma\right|
$$

like in Lemma 5.3 above for smooth elliptic curves. Since

$$
\Gamma+\Gamma^{\prime} \equiv(4 E+6 F)_{C} \quad \text { and } \quad \Gamma+p_{\Gamma} \equiv(3 E+3 F)_{C},
$$

we get $\Gamma^{\prime}-p_{\Gamma} \equiv(E+3 F)_{C}$, i.e. $\Gamma^{\prime} \equiv(E+3 F)_{C}+p_{\Gamma}$. Now, $\left|(E+3 F)_{C}+p\right|=\mid(E+$ $3 F)_{C}+p^{\prime} \mid$ if and only if $\left|(F)_{C}+p\right|=\left|(F)_{C}+p^{\prime}\right|$, which in turn is equivalent to $p=p^{\prime}$. Therefore, the linear system $L_{\Gamma}$ is uniquely defined by $p_{\Gamma}$.

A general point in $\mathbb{P}^{15}$ lies in the span of a unique divisor in each of these linear systems of degree 8. So, after projection from the point $E \cap C$, the subschemes $\Gamma$ of length 8 on $C$ whose span contains a general point $[f] \in \mathbb{P}^{14}$ in the span of $C \subseteq$ $\mathbb{P}^{14} \subseteq \mathbb{P}\left(S_{3 E+6 F}\right)$ are in one to one correspondence with linear systems $L_{\Gamma}$, and hence with the points $p_{\Gamma}$ on $C$. And the correspondence coincides with the map $\psi_{C}: \operatorname{VPS}_{C}([f], 8) \longrightarrow C$ above.

Every apolar smooth scheme $[\Gamma] \in \operatorname{VPS}_{C}([f], 8)$ spans a $\mathbb{P}^{7}$ under the embedding by $\left|(3 E+6 F)_{C}\right|$. If $[\widetilde{\Gamma}] \in \operatorname{VPS}_{C}([f], 8)$ is a limit point, then there is a $\mathbb{P}^{7}$ containing both $\widetilde{\Gamma}$ and $[f]$. If $\widetilde{\Gamma}$ spans such $\mathbb{P}^{7}$, then $\widetilde{\Gamma}$ is apolar to $f$. Otherwise, $\widetilde{\Gamma}$ spans at most a $\mathbb{P}^{6}$, implying that $C$ contains a scheme of length 8 spanning at most a $\mathbb{P}^{6}$. This is impossible: for any 6 points $P_{1}, \ldots, P_{6}$ on $C$, the subscheme $\Delta=\widetilde{\Gamma} \cup\left\{P_{1}, \ldots, P_{6}\right\}$ would span a $\mathbb{P}^{12}$, hence each hyperplane through $\Delta$ would meet $C$ in another point outside $\widetilde{\Gamma}$, so $C$ would be rational, while it is elliptic.

Every $[\Gamma] \in \operatorname{VPS}_{\Sigma}([f], 8)$ belongs to $\operatorname{VPS}_{C}([f], 8)$ for some $C \in K$, so in particular, every $[\Gamma] \in \operatorname{VPS}_{\Sigma}([f], 8)$ is apolar to $f$. Consider, therefore, the open subset $\mathcal{U}^{\prime} \subseteq \mathcal{U} \subseteq \operatorname{Hilb}_{8}(\Sigma)$ of the smooth open set $\mathcal{U}$ above consisting of schemes $\Gamma$, such that $\operatorname{dim} I_{\Gamma, 3 E+6 F}=18$, or equivalently, such that $\nu_{3 E+6 F}(\Gamma)$ spans a $\mathbb{P}^{7}$. Let $E_{\mathcal{U}}$ be the vector bundle of rank 18 over $\mathcal{U}$ whose fiber over $[\Gamma]$ is the dual of the space of sections in degree $3 E+6 F$ of the ideal $I_{\Gamma, 3 E+6 F} \subseteq T_{3 E+6 F}$. The linear form $\phi_{f, 3 E+6 F}: T_{3 E+6 F} \longrightarrow \mathbb{C}$ defines a section on $E_{\mathcal{U}}$. If $\operatorname{VPS}_{\Sigma}([f], 8) \subseteq \mathcal{U}^{\prime}$, then 
$\operatorname{VPS}_{\Sigma}([f], 8)$ is the 0 -locus of this section, since any $[\Gamma]$ in $\operatorname{VPS}_{\Sigma}([f], 8)$ is apolar to $f$.

Lemma 5.5. If $f \in S_{3 E+6 F}$ is general, then $\operatorname{VPS}_{\Sigma}([f], 8) \subseteq \mathcal{U}^{\prime}$.

Proof. It suffices to show that for any $[\Gamma] \in \operatorname{VPS}_{\Sigma}([f], 8)$ the image $\nu_{3 E+6 F}(\Gamma)$ spans a $\mathbb{P}^{7}$. But this follows from the fact that $\Gamma \subseteq C$ for some irreducible curve $C$ in $K$, and any subscheme of length 8 on the curve $\nu_{3 E+6 F}(C)$ spans a $\mathbb{P}^{7}$.

Taking all $\phi_{f, 3 E+6 F}$ for $f \in S_{3 E+6 F}$ gives a linear space of sections of $E_{\mathcal{U}}$ without basepoints on $\mathcal{U}$, so for a general $f$ the 0 -locus $\operatorname{VPS}_{\Sigma}([f], 8)$ is smooth.

Now, every point outside $\Gamma_{0}$ lies in a unique curve $C \in K$, so $\psi_{f}$ is a birational morphism from a smooth surface and has an inverse that is defined outside $\pi\left(\Gamma_{0}\right)$. Let $\pi^{\prime}: \Sigma^{\prime} \longrightarrow \mathbb{P}^{2}$ be the blowup along $\pi\left(\Gamma_{0}\right)$. Since, by assumption, all $C \in K$ are smooth at $\Gamma_{0}$, the inverse map to $\psi_{f}$ lifts to a morphism $\psi_{f}^{\prime}: \Sigma^{\prime} \longrightarrow \operatorname{VPS}_{\Sigma}([f], 8)$ that restricts to the inverse of $\psi_{C}$ on the strict transform of $\pi(C)$ on $\Sigma^{\prime}$. Therefore, $\psi_{f}^{\prime}$ is an inverse of $\psi_{f}$, and hence an isomorphism.

Acknowledgements. N. Villamizar acknowledges the support of RICAM, Linz, where she developed part of the research contained in this paper. M. Gallet would like to thank Josef Schicho and Hamid Ahmadinezhad for helpful comments, especially about the introduction. M. Gallet is supported by Austrian Science Fund (FWF): W1214-N15, Project DK9 and (FWF): P26607 and (FWF): P25652. K. Ranestad acknowledges funding from the Research Council of Norway (RNC grant 239015).

\section{References}

1. Beauville, A., Complex Algebraic Surfaces, 2nd ed., London Mathematical Society Student Texts 34, Cambridge University Press, Cambridge, 1996. Translated from the 1978 French original by R. Barlow, with assistance from N.I. Shepherd-Barron and M. Reid.

2. Catalisano, M., Geramita, A. and Gimigliano, A., Higher secant varieties of Segre-Veronese varieties, in Projective Varieties with Unexpected Properties, pp. 81-107, 2005.

3. Cox, D., The homogeneous coordinate ring of a toric variety, J. Algebraic Geom. 4 (1995), 17-50.

4. Cox, D., Little, J. and Schenck, H., Toric Varieties, Graduate Studies in Mathematics 124, Am. Math. Soc., Providence, RI, 2011.

5. Dolgachev, I., Dual homogeneous forms and varieties of power sums, Milan J. Math. 72 (2004), 163-187.

6. Fisher, T., Pfaffian presentations of elliptic normal curves, Trans. Amer. Math. Soc. 362 (2010), 2525-2540. 
7. Fogarty, J., Algebraic families on an algebraic surface, Amer. J. Math. 90 (1968), $511-521$.

8. Friedman, R., Algebraic Surfaces and Holomorphic Vector Bundles, Universitext, Springer, Berlin, 1998.

9. Fulton, W., Intersection Theory, 2nd ed., Results in Mathematics and Related Areas. 3rd Series. A Series of Modern Surveys in Mathematics 2, Springer, Berlin, 1998.

10. GaŁA̧ZCA, M., Multigraded Apolarity (2016). arXiv : 1601.06211

11. Iarrobino, A. and KAnev, V., Power Sums, Gorenstein Algebras, and Determinantal Loci, Lecture Notes in Mathematics 1721, Springer, Berlin, 1999. Appendix C by Iarrobino and Steven L. Kleiman.

12. Iliev, A. and RAnestad, K., K3 surfaces of genus 8 and varieties of sums of powers of cubic fourfolds, Trans. Amer. Math. Soc. 353 (2001), 1455-1468.

13. Kapranov, M., Chow quotients of Grassmannians. I, in I.M. Gel'fand Seminar, Adv. Soviet Math. 16, pp. 29-110, 1993.

14. Landsberg, J., Tensors: Geometry and Applications, Graduate Studies in Mathematics 128, Am. Math. Soc., Providence, RI, 2012.

15. Massarenti, A. and Mella, M., Birational aspects of the geometry of varieties of sums of powers, Adv. Math. 243 (2013), 187-202.

16. Mukai, S., Fano 3-folds, in Complex Projective Geometry, Trieste, 1989/Bergen, 1989, London Math. Soc. Lecture Note Ser. 179, pp. 255-263, 1992.

17. Oeding, L. and Ottaviani, G., Eigenvectors of tensors and algorithms for Waring decomposition, J. Symbolic Comput. 54 (2013), 9-35.

18. Ranestad, K. and Schreyer, F., Varieties of sums of powers, J. Reine Angew. Math. $\mathbf{5 2 5}$ (2000), 147-181.

19. Ranestad, K. and Schreyer, F., The variety of polar simplices, Doc. Math. 18 (2013), 469-505.

20. Ranestad, K. and Voisin, C., Variety of power sums and divisors in the moduli space of cubic fourfolds, Doc. Math. 22 (2017), 455-504.

21. Room, T., The Geometry of Determinantal Loci, Cambridge University Press, Cambridge, 1938.

22. Sylvester, J., An essay on canonical forms, supplemented by a sketch of a memoir on elimination, transformation and canonical forms, in Collected Works, vol. I, pp. 203-216, Cambridge University Press, Cambridge, 1904.

23. Sylvester, J., Sketch of a memoir on elimination, transformation, and canonical forms, in Collected Works, vol. I, pp. 184-197, Cambridge University Press, Cambridge, 1904.

Matteo Gallet

RICAM

Austrian Academy of Sciences

Altenberger Straße 69, A-4040

Linz

Austria

matteo.gallet@ricam.oeaw.ac.at
Kristian Ranestad

Department of Mathematics

University of Oslo

PO Box 1053

Blindern, N-0316

Oslo

Norway

ranestad@math.uio.no 
Nelly Villamizar

Department of Mathematics

Swansea University

Singleton Park, SA2 8PP

Swansea

U.K.

n.y.villamizar@swansea.ac.uk

Received July 18, 2016

in revised form October 27, 2016 